\title{
Description of a Non-Canonical Mn(II)-Oxidation Site in Peroxidases
}

Elena Fernández-Fueyo, ${ }^{\S \star}$ Irene Davó-Siguero, ${ }^{\S}$ David Almendral,${ }^{\S}$ Dolores Linde,${ }^{\S}$ Maria Camilla Baratto, ${ }^{\#}$ Rebecca Pogni, ${ }^{\#}$ Antonio Romero, ${ }^{\S}$ Victor Guallar, ${ }^{\text {ॠ† }}$ and Angel T. Martínez*\$

${ }^{\S}$ Centro de Investigaciones Biológicas, CSIC, Ramiro de Maeztu 9, E-28006 Madrid, Spain; "Department of Biotechnology, Chemistry and Pharmacy, University of Siena, I-53100 Siena, and Consorzio per lo Sviluppo dei Sistemi a Grande Interfase (CSGI), Florence, Italy; "Barcelona Supercomputing Center, Jordi Girona 29, E-08034 Barcelona, Spain; ${ }^{\dagger}$ ICREA, Passeig Lluís Companys 23, E-08010 Barcelona, Spain

\begin{abstract}
A dye-decolorizing peroxidase (DyP) from Pleurotus ostreatus (PosDyP4) catalyzes the oxidation of $\mathrm{Mn}^{2+}$ to $\mathrm{Mn}^{3+}$, in the presence of $\mathrm{H}_{2} \mathrm{O}_{2}$, with an efficiency similar to the well known manganese peroxidases and versatile peroxidases from this and other white-rot fungi. PosDyP4 has been overexpressed in Escherichia coli as an active enzyme, and its crystal structure has been solved at $1.56 \AA$ resolution. A combination of substrate diffusion simulations on the solved structure using the PELE software, electron paramagnetic resonance and site-directed mutagenesis led to identification of the residues involved in $\mathrm{Mn}^{2+}$ oxidation. The oxidation site in PosDyP4 is different to the conserved site in the other Mn-oxidizing peroxidases mentioned above, and it includes four acidic residues (three aspartates and one glutamate) located at the surface of the protein. Moreover, since the $\mathrm{Mn}^{2+}$ ion is not in direct contact with the heme propionates, a tyrosine residue participates in the electron transfer to the cofactor being the only essential individual residue for PosDyP4 oxidation of the metal ion. The four acidic residues contribute to $\mathrm{Mn}^{2+}$ binding in different extents, with the glutamate also involved in the initial electron transfer to the key tyrosine, as confirmed by the $>50$-fold decreased $k_{\text {cat }}$ after removing its side-chain carboxylic group. A second electron transfer pathway operates in PosDyP4 for the oxidation of aromatics and dyes starting at a surface tryptophan, as reported in other fungal and prokaryotic DyPs, and connecting with the final part of the $\mathrm{Mn}^{2+}$ oxidation route. Both tryptophanyl and tyrosyl radicals, potentially involved in catalysis, were detected by electron paramagnetic resonance of the native enzyme and its tryptophan-less variant, respectively.
\end{abstract}

KEYWORDS: Mn-oxidation site, dye-decolorizing peroxidase, crystal structure, ligand diffusion, PELE, electron transfer, QM/MM calculations, EPR spectroscopy

\section{INTRODUCTION}

Peroxidases (EC1.11.1) can oxidize a variety of substrates using peroxides as final electron acceptors. They were traditionally classified into animal and non-animal peroxidases, the second group being subdivided into three classes according to their origin, with fungal peroxidases forming class II. ${ }^{1}$ However, the recent discovery of new types of secreted fungal peroxidases has led to the establishment of two novel (super)families: the heme-thiolate peroxidases (HTP), in which classical chloroperoxidase and novel unspecific peroxygenases are included; and the dye- decolorizing peroxidases (DyP), ${ }^{2-5}$ classified together with chlorite dismutases and Escherichia coli EfeB proteins in the CDE superfamily. ${ }^{6}$

The first DyP was discovered in a Bjerkandera adusta culture described as Geotrichum candidum. ${ }^{7}$ Its crystal structure was available in $2011,{ }^{8}$ followed by that of Auricularia auricula-judae DyP. ${ }^{9}$ Their tertiary structure revealed a very different topology from the other (super)families mentioned above. Nevertheless, the DyP active site presents some similarities with class II peroxidases, such as a histidine residue as heme iron ligand, ${ }^{10}$ while a 
cysteine occupies this position in HTPs. ${ }^{2}$ However, the second histidine at the opposite side of heme in classical peroxidases is not conserved in DyPs, which generally present an aspartic acid at this position. ${ }^{5,11}$ Moreover, contrarily to class-II peroxidases, DyPs are not exclusive of fungi, with types A-C corresponding to prokaryotic enzymes and fungal DyPs preferentially included in type D. ${ }^{12}$

Since the discovery of the first DyP, a number of other family members has been studied, as wild type or heterologously expressed enzymes. ${ }^{5}$ After in vitro activation of fungal DyPs expressed as inclusion bodies, ${ }^{13}$ Pleurotus ostreatus DyP4 (PosDyP4) and a few other fungal DyPs have been recently expressed in E. coli as active proteins. ${ }^{14,15}$ Prokaryotic DyPs are easier to be expressed in E. coli facilitating different mechanistics and related studies. ${ }^{16-19}$

DyPs present a broad substrate specificity, being able to oxidize substituted phenols, azo dyes, and even lignin model compounds, among other substrates, although non-phenolic aromatics with very low activity (see Linde et al. $^{5}$ and Loncar et al. ${ }^{20}$, and references herein). Nevertheless, anthraquinonoid dyes, used in first DyP descriptions, ${ }^{7}$ appear as their best substrates. Additionally, the ability to oxidize $\mathrm{Mn}^{2+}$ to $\mathrm{Mn}^{3+}$ has been reported in DyPs from Amycolatopsis sp 75iv2 (AspDyP2), ${ }^{21} \quad P$. ostreatus (PosDyP4) ${ }^{15}$ and very recently from Irpex lacteus, ${ }^{22}$ as well as, with much lower activity levels, in a few other bacterial ${ }^{21,23-26}$ and one more fungal ${ }^{27}$ DyP. Interestingly, PosDyP4 oxidizes $\mathrm{Mn}^{2+}$ with similar efficiency to the fungal ligninolytic manganese peroxidases $(\mathrm{MnP})$ and versatile peroxidases (VP). ${ }^{15}$

Despite the variety of substrates reported, the eco-physiological role of DyPs still remains unclear. Their ability to oxidize lignin related compounds, together with their higher abundance in white-rot (ligninolytic) fungi compared with other basidiomycetes, ${ }^{5}$ and their widespread presence in forest soil fungal transcriptomes, ${ }^{28}$ support a contribution to degradation of lignin and lignin-derived compounds. Furthermore, PosDyP4 is one of the most abundant enzymes when $P$. ostreatus grows on lignocellulosic biomass. ${ }^{15}$ In the above context, DyPs have been described as bacterial "lignin peroxidases", ${ }^{29}$ although they preferentially act on phenolic technical lignins and lignin-derived substrates. ${ }^{5,30-32}$

In the present study, we identified and characterize the DyP $\mathrm{Mn}^{2+}$-oxidation site thanks to a combination of different techniques (such as X-ray diffraction, computational calculations, electron paramagnetic resonance, and directed mutagenesis). Interestingly, the site resulted to be different to the canonical $\mathrm{Mn}^{2+}$-oxidation one found in class-II peroxidases, due to its superficial location and involvement of a longrange electron pathway to heme.

\section{MATERIAL AND METHODS}

DyP Production in $\boldsymbol{E}$. coli. The PosDyP4 coding sequence (ref \#1069077 from the $P$. ostreatus genome at JGI, https://genome.jgi.doe.gov/PleosPC15_2) was cloned in the pET15b vector carrying a Nterminal His-tag sequence (Novagen, Darmstadt, Germany) and the resulting plasmid (pET15b-1069077) was used for expression in E. coli BL21(DE3)pLysS (Novagen). ${ }^{15}$ Cultures were grown for $60 \mathrm{~h}$ at $16^{\circ} \mathrm{C}$ in ZYM-5052 autoinducing medium ${ }^{33}$ in the presence of 100 $\mu \mathrm{M}$ hemin. Cells were harvested by centrifugation at $8000 \mathrm{rpm}$ for $10 \mathrm{~min}$ at $4^{\circ} \mathrm{C}$, and suspended in $100 \mathrm{~mL}$ of lysis buffer (10 $\mathrm{mM}$ Tris- $\mathrm{HCl}, \mathrm{pH} 7.5$, containing $10 \mathrm{mM}$ imidazole, and $300 \mathrm{mM} \mathrm{NaCl}$ ) supplemented with $2 \mathrm{mg} . \mathrm{mL}^{-1}$ lysozyme (Sigma-Aldrich, Steinheim, Germany) and DNase I (Roche Diagnostics, Mannheim, Germany). After $1 \mathrm{~h}$, the suspension was sonicated, debris was removed by centrifugation at 20,000 rpm for 4 $\mathrm{h}$, and the supernatant was concentrated (Amicon 10-kDa-cut-off; Millipore, Darmstadt, Germany).

The wild-type recombinant (hereinafter native) DyP and the mutated variants were purified in one single step using a 5-mL HisTag column, at a flow rate of $5 \mathrm{~mL} \cdot \mathrm{min}^{-1}$ of the above buffer in an ÄKTA HPLC system (GE Healthcare Bio Sciences AB, Uppsala, Sweden) and. After $15 \mathrm{~mL}$, the retained proteins were eluted with a $0-50 \%$ gradient of $1 \mathrm{M}$ imidazole in $50 \mathrm{~mL}$, followed by $50-100 \%$ gradient in 5 $\mathrm{mL}$ and $100 \% \mathrm{NaCl}$ in $15 \mathrm{~mL}$. Peroxidase activity was followed by 2,2-azinobis-(3ethylbenzothiazoline-6-sulfonic acid) (ABTS) oxidation, as described below. The appropriate 
fractions were pooled, concentrated and dialyzed against $10 \mathrm{mM}$ sodium tartrate ( $\mathrm{pH}$ 5).

Purification was confirmed by sodium dodecyl sulfate-polyacrylamide gel electrophoresis (SDS-PAGE) in 12\% gels stained with Coomassie brilliant blue R-250 (Sigma-Aldrich) (Figure S1), although some minor contaminating proteins were observed for some of the variants (due to the single-step purification procedure). The UV-visible spectra of native PosDyP4 and its $\mathrm{Mn}^{2+}$-oxidation site variants (Figure S2) confirmed the incorporation of heme, with characteristic Soret band at 406-407 $\mathrm{nm}$. The above agrees with: i) Reinheitszahl $\left(\mathrm{Rz}, \mathrm{A}_{\text {Soret-band }} / \mathrm{A}_{280-\mathrm{nm}}\right)$ ratios of 1.4-1.8 (except for the E345A/D352A/D354A variant with a 1.1 ratio); and ii) molar heme content, estimated by the pyridine ferrohemochrome method, ${ }^{17}$ of 0.9 for PosDyP4 and slightly lower (0.7-0.9) for the variants, due to minor contaminating proteins.

Crystal Structures. Crystallization trials were carried out by the sitting drop vapor diffusion technique, in 96-well MRC2 plates (Swissci MRC, Molecular Dimensions, Suffolk, England) using a Cartesian Honeybee System (Genomic Solutions, Irvine, USA) and the commercial screenings from Emerald (Wizard Screens I, II and III) and Jena Biosciences (JBScreen Classic Kits 1-10). Different $\mathrm{MnCl}_{2}$ concentrations were included in the crystallization trials. Drops consisted of $0.2 \mu \mathrm{l}$ of protein solution $(10 \mathrm{mg} / \mathrm{ml}$ in $10 \mathrm{mM}$ sodium tartrate, $\mathrm{pH} 5.0$ ) and $0.2 \mu \mathrm{l}$ of precipitant, and were equilibrated against $50 \mu \mathrm{l}$ reservoir solution, at $295 \mathrm{~K}$. Crystals suitable for X-ray data collection were obtained in $0.2 \mathrm{M} 2-(N-$ morpholino)ethanesulfonic acid (MES), $\mathrm{pH}$ 5.75, containing $25 \%$ polyethyleneglycol (PEG) MME 2000. Furthermore, crystal soaking in $\mathrm{MnCl}_{2}$ solutions was attempted to solve enzyme: $\mathrm{Mn}^{2+}$ structures.

Crystals were mounted in nylon loops and flash-frozen in liquid nitrogen in the mother liquor using Paratone-N (Hampton Research, Aliso Viejo, USA) as cryoprotectant. All diffraction data were collected at $100 \mathrm{~K}$ on beam line ID30B at the ESRF (Grenoble, France) using a wavelength of $0.9762 \AA$ and a Pilatus 6M detector. Diffraction data were indexed and integrated using $\mathrm{XDS},{ }^{34}$ and scaled and merged using Aimless from CCP4 program suite. $^{35}$

The structure was solved by molecular replacement using the crystal structure of $B$. adusta DyP (PDB: 3afv) as the search model and the program MOLREP in CCP4 suite. ${ }^{35}$ The final model was obtained by consecutive rounds of refinement with RefMac, in CCP4 suite, ${ }^{35}$ and alternating manual model building with $\operatorname{Coot}^{36}$ using $\sigma_{\mathrm{A}}$ weighted $2 F_{o}-F_{c}$ and $F_{o}-F_{c}$ electron density maps. Solvent molecules and MES were added automatically and visually inspected for chemically plausible positions. A total of $5 \%$ of reflections was used to calculate the $\mathrm{R}_{\text {free }}$ value throughout the refinement process. The structures were validated with MolProbity. ${ }^{37}$

$\mathrm{X}$-ray data collection and refinement statistics are summarized in Table S1. The refined model consists of a partial polypeptide chain of 484 amino acids (residues 6-489), in which the first five and the last fifteen residues were not visible in the electron density maps, one heme group, one MES and 1119 water molecules. Figures were produced with PyMOL (Schrödinger, Portland, OR, USA). The coordinates and structure factors have been deposited at the Protein Data Bank with accession code $6 \mathrm{fsk}$.

Molecular Simulations. The solved crystal structure (6fsk, chain A) was used for PosDyP4 molecular simulations. The system was prepared at $\mathrm{pH} 4.5$ (optimal activity) with the protein preparation wizard from Schrödinger, resulting in aspartic acids 32 and 241 being protonated, histidines 49, 138, 148, 168, 191 and 220 being protonated in their $\mathrm{N} \varepsilon$, and histidines 380 and 299 being double protonated (positively charged). Crystallographic water molecules were retained due to their (potential) importance in $\mathrm{Mn}^{2+}$ coordination.

For substrate diffusion molecular mechanics (MM) simulations with PELE, heme charges were obtained from a quantum mechanics/ molecular mechanics (QM/MM) minimization using QSite, ${ }^{38}$ at the DFT M06-L(lacvp*)/OPLS level of theory. Based on previous experience in manganese heme-bound systems, ${ }^{39}$ the charge of the $\mathrm{Mn}^{2+}$ ion was set to 1.2. The ion was then placed on five different initial positions, all of them in the bulk solvent at distances $>25 \AA$ from the heme iron. The new adaptive-PELE software ${ }^{40}$ was used to study $\mathrm{Mn}^{2+}$ diffusion and 
binding. The adaptive protocol improves sampling in PELE by running multiple short simulations (epochs) where the initial conditions in each of them are selected through a reward function aiming at sampling non-visited areas. In particular, three simulations of 256 processors with 20 epochs, of twelve PELE steps each, were used. We should remark that along all simulations, the ligand moves freely, without any predetermined bias. Interaction energies, in $\mathrm{kcal} / \mathrm{mol}$, were derived as Eab - (Ea $+\mathrm{Eb})$ where Eab is the total energy of the complex, Ea the energy of the "receptor" (protein + crystallographic waters), and Eb the energy of the $\mathrm{Mn}^{2+}$ ion; all of them obtained at the OPLS2005 level of theory with a surface GB implicit solvent model.

Oxidation QM/MM simulations were performed also with Qsite. ${ }^{38}$ The QM region included: the heme (compound I, i.e. $\mathrm{Fe}^{4+}=\mathrm{O}$ and porphyrin cation radical complex), Trp405, $\mathrm{Mn}^{2+}$ ion, residues 215,334 to 345 and 352 to 357 , and two water molecules coordinated to the metal ion, for a total of $502 \mathrm{QM}$ atoms. They were modeled at the DFT M06-L(lacvp*) level of theory, the reminder of the system being described with the OPLS-AA force field. To facilitate convergence of compound-I's electronic wave function, the triplet spin state was selected. ${ }^{41}$ For the QM/MM e-pathway calculations, ${ }^{42}$ monitoring the electron transfer route, the heme group, $\mathrm{Mn}^{2+}$ ion and its directly coordinated residues were subtracted from the QM region. Three iterations of the procedure were performed, where Trp405 and both Trp405 and Tyr339 were subtracted from the QM region in the second and third iterations, respectively.

Electron paramagnetic resonance (EPR) Measurements. Continuous-wave EPR X-band $(9.38 / 9.39 \mathrm{GHz})$ measurements were performed with a Bruker E500 Elexsys Series using the Bruker ER 4122SHQE cavity, equipped with an Oxford Instruments helium continuous flow cryostat (ESR900). Enzyme activation was carried out by adding $\mathrm{H}_{2} \mathrm{O}_{2}$ in a PosDyP4/peroxide molar ratio of $1: 8$, with a final concentration of $0.25 \mathrm{mM}$ enzyme in tartrate buffer, $\mathrm{pH} 3 . \mathrm{H}_{2} \mathrm{O}_{2}$ was added directly in a $3.5 \mathrm{~mm}$ EPR tube, and the reaction time before freezing was $\sim 10 \mathrm{~s}$. The $g$-values were calculated with a strong pitch sample as reference, and an error of \pm 0.0002 was estimated.

Site-Directed Mutagenesis. The D196A, D215V, Y339A, E345A, D352A, D354A and W405S simple mutations, and the D352A/ D354A double and E345A/D352A/D354A triple mutations were introduced in the PosDyP4 sequence by PCR using the expression plasmid pET15b-1069077 (see above) as template, and the QuikChange kit from Stratagene (La Jolla, CA, USA). The direct and reverse primers for PCR (Table S2) were synthesized. To obtain the triple variant, the plasmid harboring the D352A/D354A mutations was used as a template and combined with the primer E345A.

The PCR reaction ( $50 \mu$ l volume) was carried out in an Eppendorf (Hamburg, Germany) Mastercycler pro-S thermal cycler using $20 \mathrm{ng}$ of template DNA, $500 \mu \mathrm{M}$ each dNTP, $125 \mathrm{ng}$ direct and reverse primers, 2.5 units of PfuTurbo polymerase (Stratagene), and the manufacturer's buffer. Reaction conditions included: i) a start cycle of $1 \mathrm{~min}$ at $95^{\circ} \mathrm{C}$; ii) 18 cycles of $50 \mathrm{~s}$ at $95^{\circ} \mathrm{C}, 50 \mathrm{~s}$ at $55^{\circ} \mathrm{C}$, and $10 \mathrm{~min}$ at $68^{\circ} \mathrm{C}$; and iii) a final cycle of $10 \mathrm{~min}$ at $68^{\circ} \mathrm{C}$. The mutated genes were expressed in E. coli and the variants purified as described above for the wild gene and native DyP.

Enzyme Kinetics. The kinetic constants of PosDyP4 and its variants were estimated from absorbance changes during substrate oxidation in $0.1 \mathrm{M}$ tartrate (optimal $\mathrm{pH}$ values) at $25^{\circ} \mathrm{C}$ in a Biomate5 spectrophotometer (Thermo Scientific). The reactions were initiated by addition of $\mathrm{H}_{2} \mathrm{O}_{2}$ to a concentration of $0.25 \mathrm{mM}$.

Oxidation of $\mathrm{Mn}^{2+}$ was followed at $\mathrm{pH} 4.5$ by monitoring formation of $\mathrm{Mn}^{3+}$-tartrate complex $\left(\varepsilon_{238} 6.5 \mathrm{mM}^{-1} . \mathrm{cm}^{-1}\right)$. Reactive Blue 19 (RB19) and ABTS oxidation were assayed at $\mathrm{pH} 3.5$, and monitored by RB19 disappearance $\left(\varepsilon_{595} 10\right.$ $\mathrm{mM}^{-1} \cdot \mathrm{cm}^{-1}$ ) and formation of ABTS cation radical ( $\varepsilon_{436} \quad 29.3 \quad \mathrm{mM}^{-1} . \mathrm{cm}^{-1}$ ), respectively. Reactive Black 5 (RB5), 2,6-dimethoxyphenol (DMP) and veratryl alcohol (VA) oxidation were assayed at $\mathrm{pH} 3$, and monitored by RB5 disappearance $\left(\varepsilon_{598} \quad 30 \mathrm{mM}^{-1} \cdot \mathrm{cm}^{-1}\right)$, dimeric coerulignone $\left(\varepsilon_{469} 55 \mathrm{mM}^{-1} . \mathrm{cm}^{-1}\right)$ formation, and veratraldehyde $\left(\varepsilon_{310} 9.3 \mathrm{mM}^{-1} . \mathrm{cm}^{-1}\right)$ formation, respectively.

Means and standard errors for Michaelis constant $\left(K_{\mathrm{m}}\right)$ and turnover $\left(k_{\text {cat }}\right)$ values were 
obtained by nonlinear least-squares fitting to the Michaelis-Menten model. Fitting of these constants to the normalized equation $v=$ $\left(k_{\mathrm{cat}} / K_{\mathrm{m}}\right)[\mathrm{S}] /\left(1+[\mathrm{S}] / K_{\mathrm{m}}\right)$ yielded the catalytic efficiencies $\left(k_{\text {cat }} / K_{\mathrm{m}}\right)$ with their standard errors. Biphasic kinetics was observed for $\mathrm{Mn}^{2+}$ oxidation by some PosDyP4 variants enabling calculation of two sets of kinetic constants, as found also for other DyPs. ${ }^{13,43}$

\section{RESULTS AND DISCUSSION}

Solving the Crystal Structure of PosDyP4. We have solved the crystal structure of PosDyP4 at $1.56 \AA$ resolution, after the heterologous expression in E. coli, as an active enzyme, of the sequence obtained from the $P$. ostreatus genome available at the DOE JGI. ${ }^{15}$ All attempts to solve the structure of native PosDyP4 in complex with $\mathrm{Mn}^{2+}$ were unsuccessful (an electron-density map, corresponding to the Asp215, Tyr339, Glu345, Asp352, Asp354 and water31 region discussed below, is shown in Figure S3). PosDyP4 shows the overall topology (Figure 1A) characteristic of the DyP family, and other members of the CDE superfamily. ${ }^{5,6}$ Each of the two ferredoxinlike domains is formed by a four-stranded antiparallel $\beta$-sheet (strands B1-B6 and B7-B11 in the $\mathrm{C}$-terminal and $\mathrm{N}$-terminal domains, respectively) and several helices (up to a total of twenty-eight, when short helical regions are included). Two additional $\beta$-strands (B9 and B10) are located at the bottom of the N-terminal domain, which also harbors the heme cofactor.

A structural search with the PosDyP4 molecular structure at the DALI server ${ }^{44}$ shows the highest fitting ( $\mathrm{Z}$ value) and identity (id) with the reported crystal structures of: i) the only two fungal DyPs whose structure had been reported, from $B$. adusta and Auricularia auricula-judae (Z>51, id $>43 \%)$; ii) two structurally-related DyPs from Amycolatopsis sp and Anabaena sp (Z:39, id:28-29\%); and iii-iv) two other groups of prokaryotic DyPs and CDEproteins with eleven (Z:26-28, id:15-20\%) and

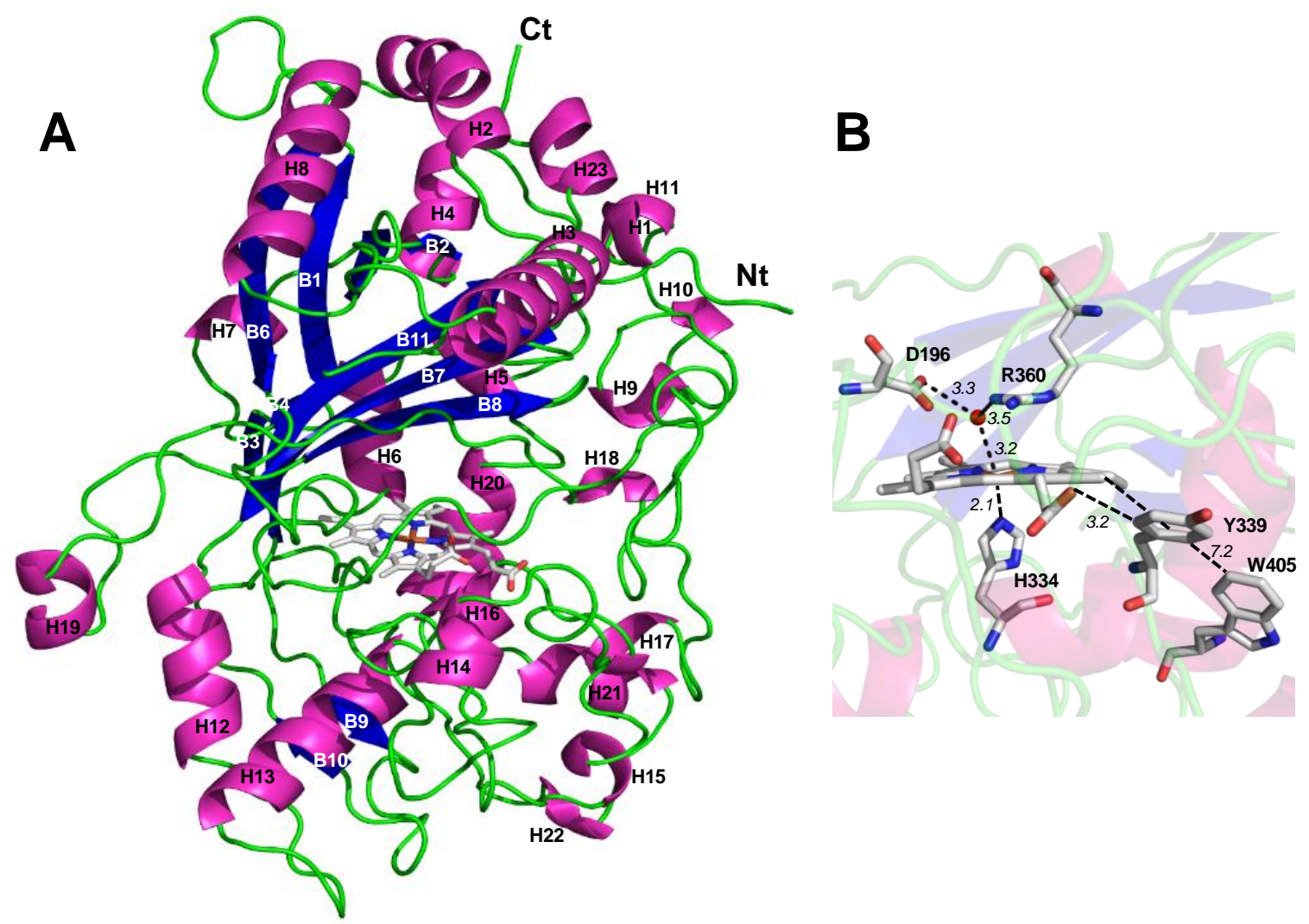

Figure 1. PosDyP4 crystal structure. A) Ribbon representation of the overall fold with $\alpha$-helices in magenta ( $\mathrm{H} 1$ to $\mathrm{H} 28), \beta$-strands in blue forming two $\beta$-sheets (B1-B6 and B7, B8 and B11), random coil in green, $\mathrm{C}$ and $\mathrm{N}$ termini of the solved structure $(\mathrm{Ct}$ and $\mathrm{Nt})$ included, and the heme cofactor as CPK-colored sticks (PDB: 6fsk). B) Detail of the heme pocket with His334, Asp196, Arg360 and water134 (at the sixth coordination position of heme iron), and Trp405 and Tyr339 at 7.2 and $3.2 \AA$ from the heme cofactor, respectively, as CPK-colored sticks. 
two (Z:12, id:7-9\%) members (see Table 1 with PDB entries and additional details).

Although the sequence identity is low, around $50 \%$ among fungal members and below $30 \%$ between the fungal and prokaryotic members, there are noteworthy similarities in the tertiary structure of the above DyPs and related CDE proteins (Figure S4). A peculiarity of the P. ostreatus, Amycolatopsis $\mathrm{sp}$ and Anabaena sp DyPs is a prominent helix-8 (red asterisks in Figure S4). ${ }^{12,21}$ Interestingly, it has been proposed to include these two prokaryotic proteins in a new DyP type together with the fungal DyPs. ${ }^{45}$ AspDyP2 and PosDyP4 also share its ability to efficiently oxidize $\mathrm{Mn}^{2+}$, as described below.

In contrast with class-II peroxidases, that have a main heme access channel (in front of the heme $\delta$-meso carbon) and eventually a small second channel in front of one of the heme propionates $\left(\mathrm{Mn}^{2+}\right.$ channel $){ }^{10}$ PosDyP4 only has a narrow access channel connecting the solvent with the upper side of the heme pocket (Figure S5) and lacks a channel at the vicinity

\begin{tabular}{|c|c|c|c|c|}
\hline & PDB & $\AA$ & Z & $\%$ \\
\hline Bjerkandera adusta & 3afv & 1.4 & 54 & 48 \\
\hline Auricularia auricula-judae & 4au9 & 2.1 & 51 & 43 \\
\hline Amycolaptosis sp & $4 \mathrm{~g} 2 \mathrm{c}$ & 2.3 & 39 & 28 \\
\hline Anabaena sp & $5 c 2 i$ & 1.9 & 39 & 29 \\
\hline Thermobifida fusca & $5 f w 4$ & 1.8 & 28 & 20 \\
\hline Shewanella oneidensis & 2iiz & 2.3 & 28 & 17 \\
\hline Escherichia coli & 3072 & 2.0 & 28 & 20 \\
\hline Thermomonospora curvata & $5 j \times u$ & 1.8 & 28 & 18 \\
\hline Streptomyces lividans & $5 \mathrm{mjh}$ & 1.5 & 27 & 19 \\
\hline Streptomyces coelicolor & 4gt2 & 1.8 & 27 & 19 \\
\hline Bacteroides thetaiotaomicron & 2gvk & 1.6 & 27 & 16 \\
\hline Enterobacter ligninolyticus & $5 v j 0$ & 1.9 & 26 & 28 \\
\hline Rhodococus jostii & 3veg & 2.4 & 27 & 15 \\
\hline Thermobifida cellulosilytica & 4gs1 & 1.7 & 26 & 19 \\
\hline Vibrio cholera & $5 \mathrm{de} 0$ & 2.2 & 26 & 19 \\
\hline Candidatus Nitrospira difluvii & $3 n n 2$ & 1.9 & 12 & 9 \\
\hline Thermus thermophilus & $1 \mathrm{vdh}$ & 2.0 & 12 & 7 \\
\hline
\end{tabular}

${ }^{\mathrm{a}} \mathrm{PDB}$ entries, resolution $(\AA), \mathrm{Z}$ value and identity with PosDyP4 (\%) are provided of the heme propionates. This resulted in the exposed $\mathrm{Mn}^{2+}$-oxidation site described here.

The heme pocket region in PosDyP4 (Figure 1B) shows several conserved residues including His334, which appears as a ligand of the heme iron, and Asp196 occupying a mirror position at the opposite side of the cofactor together with neighboring Arg360, and Trp405 and Tyr339 mentioned below at $\sim 7$ and $\sim 3 \AA$ from the heme, respectively. Asp196 and Trp405 are homologous to residues involved in catalysis by other fungal DyPs. ${ }^{5}$ To confirm their role in PosDyP4, they were substituted by alanine and serine, respectively, and the activities (on ABTS, DMP, $\mathrm{Mn}^{2+}$, RB5, RB19 and VA) were analyzed on different substrates (data not shown in tables). Asp196, located over the heme plane, is essential in PosDyP4, since no activity was detected in the D196A variant. This is in agreement with a role in the heterolytic cleavage of $\mathrm{H}_{2} \mathrm{O}_{2}$ to form compound $\mathrm{I}$, as reported in other fungal $^{5,11}$ and some bacterial DyPs. ${ }^{27}$

Mutation of the solvent-exposed Trp405 (W405S variant) suppressed VA oxidation and decreased the activity towards all other substrates with the only exception of $\mathrm{Mn}^{2+}$, whose oxidation was unaffected. This tryptophan would be homologous to AauDyP Trp377 involved in oxidation of high redoxpotential substrates, such as VA, by formation of a solvent-exposed aromatic radical, followed by long-range electron transfer from the substrate to the heme. ${ }^{43}$ Formation of a similar catalytically-active tryptophanyl radical has been recently demonstrated also for Thermomonospora curvata $\mathrm{DyP},{ }^{18}$ while reactive tyrosyl radicals have been reported in other prokaryotic DyPs. ${ }^{46}$ Interestingly, the Trp405 radical was detected by EPR (see below) after peroxide activation of PosDyP4, and the W405S variant was useful in EPR experiments, as described below.

Diffusion Simulations Reveal an Unusual $\mathrm{Mn}^{2+}$ Binding Site. Attempts to obtain the crystal structure of a PosDyP4: $\mathrm{Mn}^{2+}$ complex failed. Therefore, to identify the $\mathrm{Mn}^{2+}$-oxidation site, a free (non-biased) exploration of $\mathrm{Mn}^{2+}$ diffusion with adaptive PELE ${ }^{40}$ was performed mapping the whole enzyme surface. Figure 2 shows the protein-ligand interaction energy profile against the distance from the $\mathrm{Mn}^{2+}$ ion to the Asp354 mentioned below. 


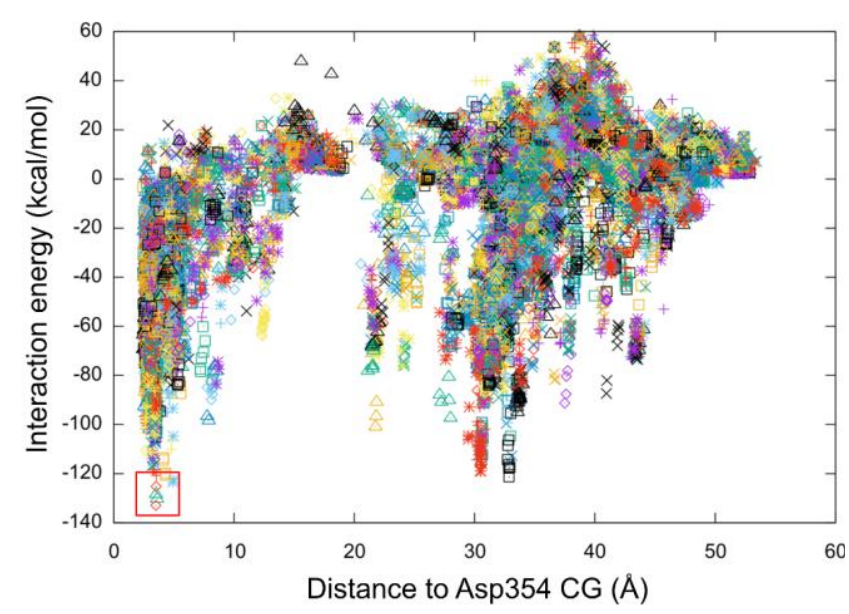

Figure 2. Results of $\mathrm{Mn}^{2+}$ diffusion with adaptivePELE. The protein-ligand interaction energy is shown against the distance to the side-chain carboxyl CG atom in Asp354. Each color (symbol) corresponds to a different trajectory. The best energy positions were located near Asp354 (red box) and one of them is shown in Figures 3 and 4.

While the ion finds multiple small cavities (local minima) in various sites of the surface, all simulations indicated a highly-acidic area (Figure 3) where $\mathrm{Mn}^{2+}$ binds preferentially coordinated by the Glu345, Asp352 and Asp354 carboxyl groups at $\sim 2 \AA$ distance (Figure 4). These three acidic residues, along with Asp215 (at a longer distance from the $\mathrm{Mn}^{2+}$ ion) and two water molecules, form an octahedral coordination environment. Tyr339, another

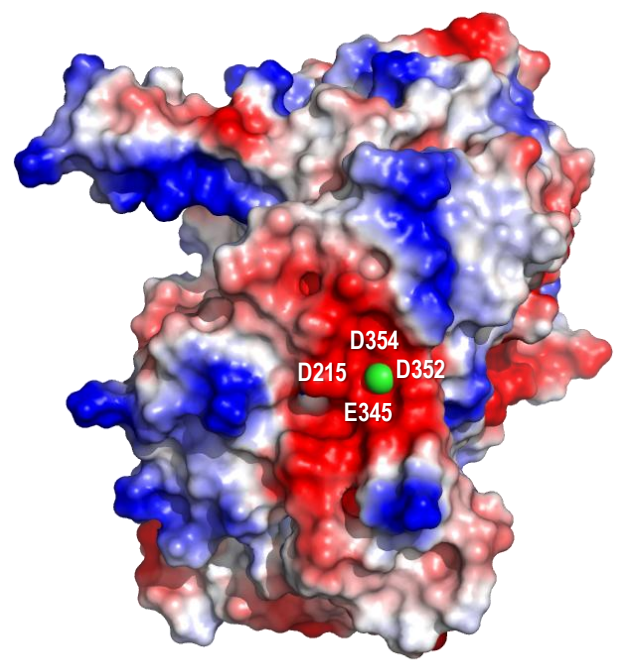

Figure 3. Solvent access surface of PosDyP4, colored by electrostatic potential (blue, positive; and red, negative) showing the highly acidic region (with Asp215, Glu345, Asp352 and Asp354) surrounding the $\mathrm{Mn}^{2+}$ ion (green sphere) after its diffusion by PELE (on PDB: 6fsk).

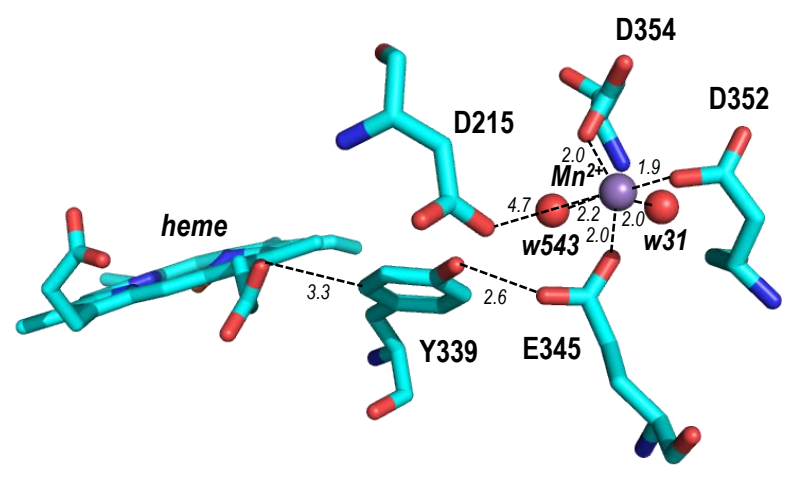

Figure 4. Four acidic residues and two water molecules (red spheres) involved in the coordination of $\mathrm{Mn}^{2+}$ (purple sphere) by PosDyP4, and Tyr339 located between the $\mathrm{Mn}^{2+}$ binding site and the heme cofactor (residues and heme as CPK-colored sticks), in one snapshot from the best ligand-binding region in PELE simulations (red square in Figure 3). Some distances are indicated in $\AA$.

residue involved in PosDyP4 oxidation of $\mathrm{Mn}^{2+}$, as shown by site-directed mutagenesis, is far from the metal ion $(4.9 \AA)$ but at $2.6 \AA$ from Glu345 (Figure 4) bridging the $\mathrm{Mn}^{2+}$-binding site with the heme cofactor. Comparison between the lowest interaction energy structure and the (apo) crystal structure without $\mathrm{Mn}^{2+}$ reveals some side-chain rearrangement in several amino acids during binding of the metal ion (Figure S6).

The Electron Transfer Route was Mapped by QM/MM Simulations. Figure $\mathbf{5 A}$ shows the total spin density obtained in QM/MM modeling of the $\mathrm{Mn}^{2+}$ oxidation process, with over 500 atoms being included in the quantum calculations. As in previous models of cytochromes's compound $\mathrm{I}^{41}$ we observe two unpaired electrons in the iron-oxo moiety, localized in the $d_{x z} / d_{y z}$ and $p_{x} / p_{y}$ orbitals, respectively. More relevant is the nature of the third unpaired electron, largely localized in the $\mathrm{Mn}^{2+} \mathrm{d}$ orbitals. This indicates that compound I is capable of extracting one electron from the $\mathrm{Mn}^{2+}$ ion. In addition, we observe a minor component on Trp405, supporting the role of this residue as a catalytic radical, as shown by EPR and directed mutagenesis.

Figure 5B shows the results of applying the $\mathrm{QM} / \mathrm{MM}$ e-pathway procedure, where we aim at mapping the electron transfer pathway. In this type of simulations we do not include the donor and acceptor (heme and $\mathrm{Mn}^{2+}$ ) in the $\mathrm{QM}$ region, focusing instead in the transfer region. ${ }^{42}$ 

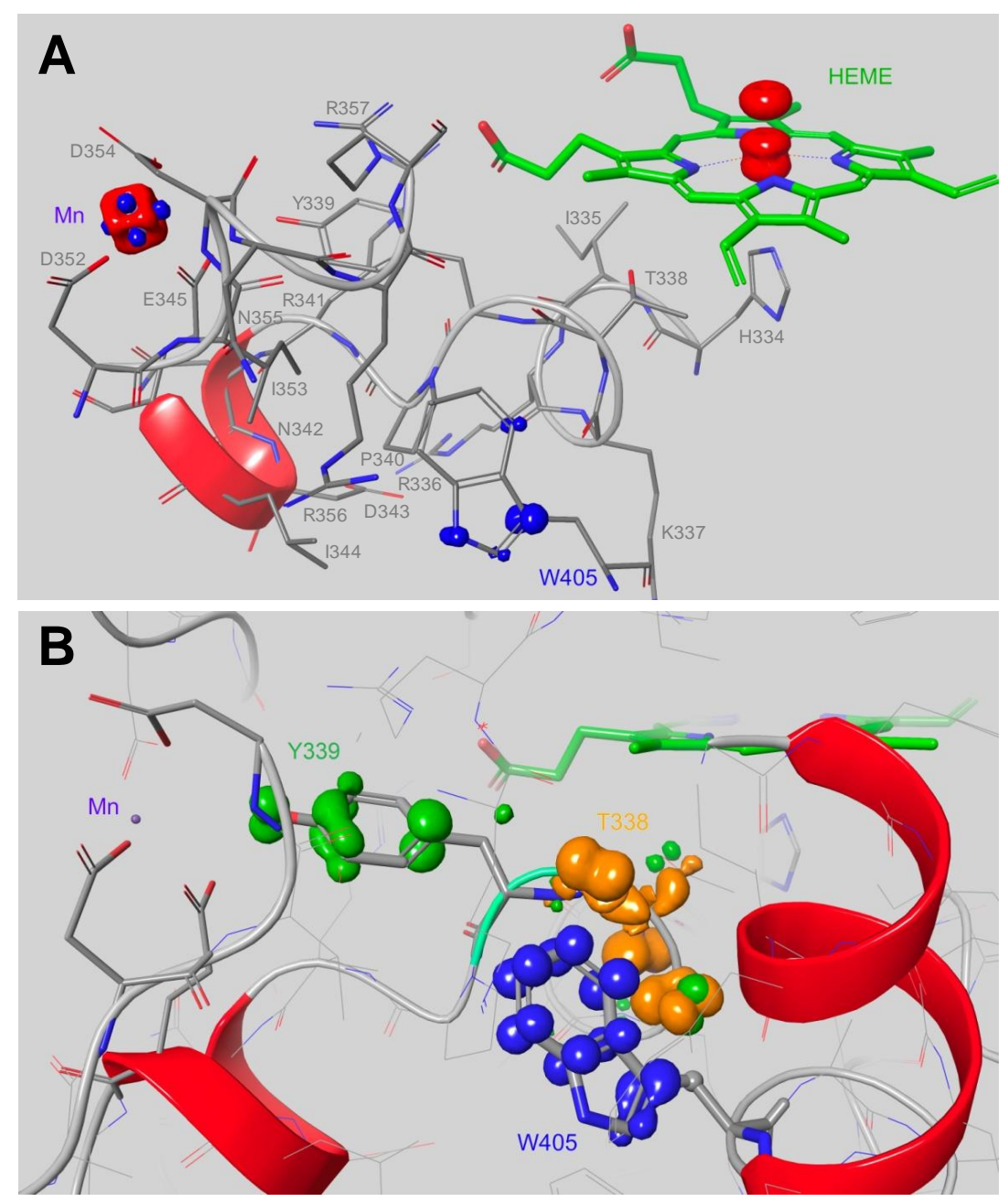

Figure 5. QM/MM simulations of $\mathrm{Mn}^{2+}$ oxidation and electron transfer to heme. A) Spin density in the $\mathrm{QM} / \mathrm{MM}$ calculations for $\mathrm{Mn}^{2+}$ oxidation in the triplet state. Residues in the $\mathrm{QM}$ region are shown in thin sticks, together with backbone trace in a cartoon style. The heme is shown in green and a thicker stick format. B) Electron transfer route from the QM/MM e-pathway analysis. The spin densities of the first three iterations are shown (in order): Trp405 (blue), Tyr339 (green) and Thr338 (orange). These three regions, and the environment of the donor $\left(\mathrm{Mn}^{2+}\right)$ and acceptor (heme) are shown in sticks; the remainder of the system is in wire style. The enzyme backbone is shown in a cartoon style.

The results show the first three iterations of the procedure: Trp405 in blue, Tyr339 in green and Thr338 in orange. Thus, the analysis indicates that the $\mathrm{Mn}^{2+}$ oxidation and the abovementioned Trp405 electron transfer pathways might converge in the backbone of Thr338, which is located next to the axial histidine. A close look at the carbonyl backbone of this threonine indicates a large hydrophobic environment, raising the energy levels of its $\pi$ orbitals and facilitating the electron transport.

Protein Radical Formation was Shown by EPR. In the EPR spectrum of the native PosDyP4 resting state, the heme $\mathrm{Fe}^{3+}$ is in a rhombically distorted high spin state with an almost undetectable amount of low spin state (Figure S7, black line). After the addition of
$\mathrm{H}_{2} \mathrm{O}_{2}$ (Figure S7, red line) the $\mathrm{Fe}^{3+}$ signal decreased and the appearance of a radical species was observed, a close up of which is shown in Figure 6A. The lineshape and the $g$ value equal to 2.0041 are similar to that reported for the $\mathrm{H}_{2} \mathrm{O}_{2}$-activated AauDyP, which was assigned to a tryptophanyl radical in combination with other minor radical species. $^{43,47}$

Radicals immobilized in proteins are mainly represented by tryptophanyl and/or tyrosyl radicals, whose identification and assignment might lead to ambiguities. In both cases the dominant splitting in the EPR spectrum arises from distinct hyperfine couplings of the $\beta$ methylene protons $\beta_{1}$ and $\beta_{2}$ at $C_{\beta}$, attached to $\mathrm{C} 3$ or $\mathrm{C} 1$ of the tryptophan or tyrosine ring, 


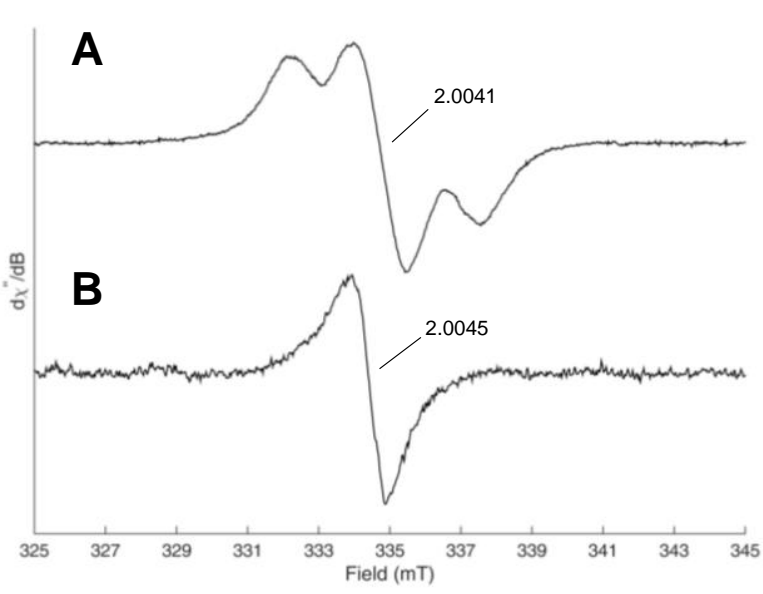

Figure 6. $20 \mathrm{mT}$ scan width X-band EPR spectra of $0.25 \mathrm{mM}$ native PosDyP4 (A) and W405S variant (B) in tartrate buffer, $\mathrm{pH} 3$, after the addition of 8 eq. of $\mathrm{H}_{2} \mathrm{O}_{2}$. Conditions were $5 \mathrm{~mW}$ microwave power, $0.2 \mathrm{mT}$ modulation amplitude, and $v=9.39$ (A) or 9.38 (B) GHz. The EPR spectra are normalized (see Figures S7 and S8 for whole spectrum, before and after $\mathrm{H}_{2} \mathrm{O}_{2}$ addition, and for comparison of nonnormalized signals, respectively).

respectively. ${ }^{47}$ The hyperfine coupling constants of these two $\beta$ protons govern the EPR spectrum and depend from the side-chain orientation measured as the dihedral angle $(\vartheta)$ for each of the $\beta$ protons according to the HellerMcConnell relation. ${ }^{47}$ When the dihedral angles are equivalent the side chain is either in the tryptophan ring plane or perpendicular to it. In the first case, the dihedral angle for both $\beta$ protons is $\approx 30^{\circ}$ and in the latter case $\approx 60^{\circ}$.

The crystallographic dihedral angles for the $\beta$ protons of $\operatorname{Trp} 405$ (PDB: 6fsk) are $\vartheta_{1} \approx\left|23.5^{\circ}\right|$ for $H-\beta_{1}$ and $\vartheta_{2} \approx \mid 35.5^{\circ}$ for $H-\beta_{2}$. These values correspond to almost equivalent isotropic coupling constants $\left(2.18 \mathrm{mT}\right.$ for $\mathrm{H}-\beta_{1}$ and 1.72 $\mathrm{mT}$ for $\mathrm{H}-\beta_{1}$, calculated from the HellerMcConnell relation) and are in agreement with the $\mathrm{A}_{\text {iso }}=1.92 \mathrm{mT}$ value determined from the experimental spectrum where the tryptophanyl radical represents the main contribution. For AauDyP the crystallographic dihedral angles for the $\beta$ protons are $\vartheta_{1} \approx \mid 30^{\circ}$ for $\mathrm{H}-\beta_{1}$ and $\vartheta_{2} \approx\left|40^{\circ}\right|$ for $\mathrm{H}-\beta_{2}$ revealing approximately the same orientation for both the catalytically active Trp405 and Trp377 in PosDyP4 and AauDyP, respectively. ${ }^{43,47}$

The EPR spectrum of the W405S variant, which was shown to be inactive on all the substrates assayed except on $\mathrm{Mn}^{2+}$, also shows a high-spin $\mathrm{Fe}^{3+}$. After the addition of $\mathrm{H}_{2} \mathrm{O}_{2}$, a low intensity signal of a protein radical species is observed, lacking the tryptophanyl radical component observed in the native PosDyP4 spectrum (Figures 6B and S8). The nonstructured signal has a linewidth around $1 \mathrm{mT}$, and its $g$ value (2.0045) suggests a tyrosyl radical, similar to those detected in the $\mathrm{H}_{2} \mathrm{O}_{2-}$ activated form of several peroxidases. ${ }^{43,47,48}$

The PosDyP4 crystal structure shows Tyr339, and several phenylalanine residues, in close proximity to Trp405. Therefore, the mixed protein radical in the EPR spectrum of activated PosDyP4 includes components of both Trp405 (its main contribution being also confirmed by the spectrum of the W405S variant lacking the tryptophanyl component) and Tyr339 radicals. Then, the tyrosyl signal observed in the EPR spectrum of the W405S variant most probably corresponds to Tyr339, whose radical signal is affected by the neighboring phenylalanine residues.

These EPR results substantiate the QM/MM calculations that: i) identified Trp405 as the residue with the highest tendency to form a radical (highest spin density) at the heme neighborhood (Figure 5A); and ii) predicted that this tendency would pass to Tyr339 in the W405S variant, as shown when excluding Trp405 from the QM region (Figure 5B).

Mutagenesis Confirms the Functionality of the $\mathrm{Mn}^{2+}$-oxidation Site. According to the computational predictions, four acidic amino acids (Asp215, Glu345, Asp352 and Asp354) could be involved in the binding of $\mathrm{Mn}^{2+}$, with Tyr339 also located at the cation vicinity (Figure 4). To confirm the role of the above residues, we replaced them for alanines and seven mutated variants, carrying from 1 to 3 mutations, were characterized.

As shown in Table 2, all the variants exhibited a reduced $\mathrm{Mn}^{2+}$ oxidation activity, while their activity on ABTS (which initially was only slightly lower than the one found on $\mathrm{Mn}^{2+}$ ) remained basically unchanged (Table S3). This confirms the specific role of these residues in $\mathrm{Mn}^{2+}$ binding and oxidation. Tyr339 appears as the most important residue since no $\mathrm{Mn}^{2+}$ oxidation was observed for the Y339A variant. Glu345 seems to be also a key residue, since its E345A variant exhibited a 50-fold 
Table 2. Steady-state Kinetic Parameters - $K_{\mathrm{m}}(\mu \mathrm{M}), k_{\mathrm{cat}}\left(\mathrm{s}^{-1}\right)$ and $K_{\mathrm{m}} / k_{\mathrm{cat}}\left(\mathrm{s}^{-1} \cdot \mathrm{mM}^{-1}\right)$ - for Oxidation of $\mathbf{M n}^{2+}$ by PosDyP4 and Seven Variants Mutated at the $\mathrm{Mn}^{2+}$-Oxidation Site (with Two Sets of Constants in Three of Them, Characterized by Higher and Lower Affinity for $\left.\mathrm{Mn}^{2+}\right)^{\mathrm{a}}$

$\begin{array}{clcccccccc} & & \text { DyP4 } & \text { Y339A } & \text { D215A } & \text { E345A } & \text { D352A } & \text { D354A } & \begin{array}{c}\text { D352A } \\ \text { D354A }\end{array} & \begin{array}{c}\text { E345A/ } \\ \text { D352A/ } \\ \text { D354A }\end{array} \\ \begin{array}{clccccccc}\text { Mn }^{2+} \\ \text { Higher Affinity }\end{array} & K_{\mathrm{m}} & 290 \pm 30 & - & 4400 \pm 1000 & 630 \pm 90 & 350 \pm 120 & 260 \pm 10 & 300 \pm 40 & 1400 \pm 160 \\ & k_{\text {cat }} & 56 \pm 2 & 0 & 28 \pm 2 & 1 \pm 0 & 13 \pm 2 & 10 \pm 0 & 25 \pm 1 & 11 \pm 0 \\ & k_{\text {cat }} / K_{m} & 200 \pm 20 & 0 & 6 \pm 0 & 2 \pm 0 & 36 \pm 0 & 39 \pm 1 & 85 \pm 5 & 8 \pm 0 \\ M^{2+} & K_{m} & - & - & - & - & 3500 \pm 900 & 4300 \pm 700 & 2300 \pm 700 & - \\ \text { Lower Affinity } & k_{\text {cat }} & 0 & 0 & 0 & 0 & 40 \pm 3 & 39 \pm 2 & 73 \pm 5 & 0 \\ & k_{\text {cat }} / K_{m} & 0 & 0 & 0 & 0 & 11 \pm 1 & 9 \pm 1 & 32 \pm 4 & 0\end{array}$

${ }^{\mathrm{a}}$ Means and $95 \%$ confidence limits from reactions at $25^{\circ} \mathrm{C}$ in $0.1 \mathrm{M}$ tartrate, $\mathrm{pH} 4.5$.

decrease in the $k_{c \text { at }}$ and a 2 -fold increase in the $K_{\mathrm{m}}$, resulting in an overall 100 -fold lower catalytic efficiency (Table 2 and Figure 7). In contrast, substitution of Asp215, Asp352 or Asp354 had a lesser effect in terms of $\mathrm{Mn}^{2+} k_{\text {cat }}$, which was less than 2-fold reduced, most probably due to suboptimal positioning of the substrate ion.

Nevertheless, changing the latter three residues impaired the $\mathrm{Mn}^{2+}$ affinity, resulting in an increase of the $K_{\mathrm{m}}$ value from 9- to 15-fold. Interestingly, the Asp352 and Asp354 substitutions, individually or together, resulted in a biphasic kinetics, as shown in Figure 7 (see Figure S9 for representation using a nonlogarithmic scale). These results enabled calculation of two sets of kinetic constants, as described previously, ${ }^{13,43}$ characterized by their higher and lower affinities for $\mathrm{Mn}^{2+}$ (which would correspond to different binding sites or geometries) (Table 2). The combination of mutations (D352A/D354A or E345A/D352A/ D354A) strongly impaired the enzyme activity, but no $\mathrm{Mn}^{2+}$ oxidation was only found for the Y339A variant.

A New $\mathrm{Mn}^{2+}$-Oxidation Site Exists in DyPs. The well characterized $\mathrm{Mn}^{2+}$ oxidation site in fungal MnPs and VPs comprises three acid residues (Glu35, Glu39 and Asp179 in Phanerochaete chrysosporium MnP, PDB: 1mnp; and Glu36, Glu40 and Asp175 in Pleurotus eryngii VP, PDB: 2boq) and one heme propionate. ${ }^{10,49}$ This site includes a narrow access channel towards the heme propionate or manganese channel, different from the wider heme access channel that provides access to $\mathrm{H}_{2} \mathrm{O}_{2}$ and some reducing substrates to the heme edge. ${ }^{10}$ In the above channel, the $\mathrm{Mn}^{2+}$ ion is located at $\sim 2 \AA$ from the heme propionate, allowing direct electron transfer. However, in PosDyP4, the $\mathrm{Mn}^{2+}$ binding site is located at the protein surface further from the heme $(\sim 11 \AA)$, and an electron transfer pathway is required. This pathway involves Tyr339, as predicted by the QM/MM calculations and confirmed by the mutagenesis experimental results described above.

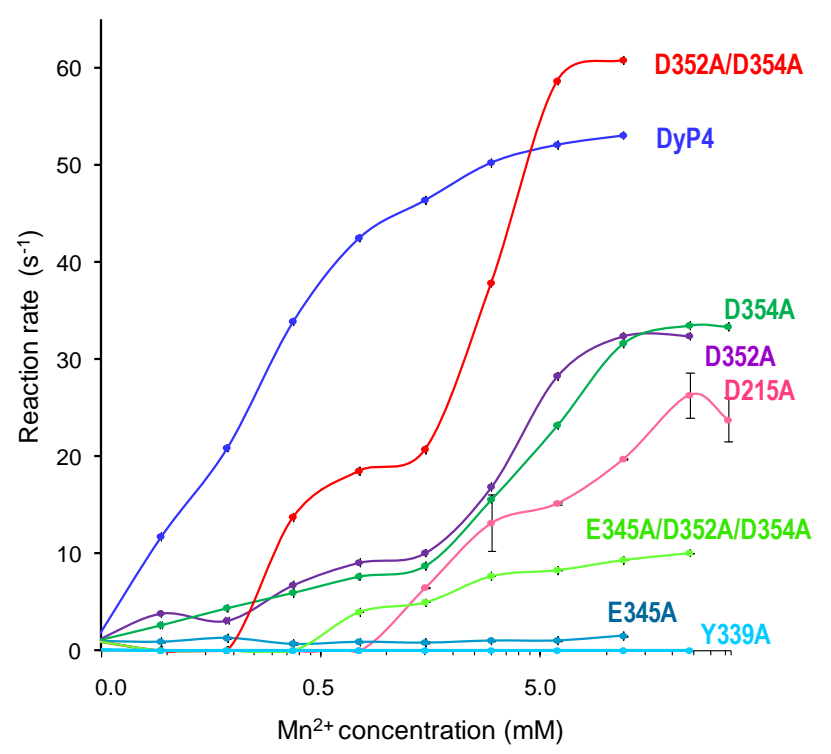

Figure 7. Kinetics of $\mathrm{Mn}^{2+}$ oxidation by PosDyP4 and seven simple, double and triple variants (substrate concentration in a logarithmic scale). Biphasic kinetics are evident for several variants, such as D352A/D354A, compared with native DyP4 (see Table 2). Means and 95\% confidence limits. 


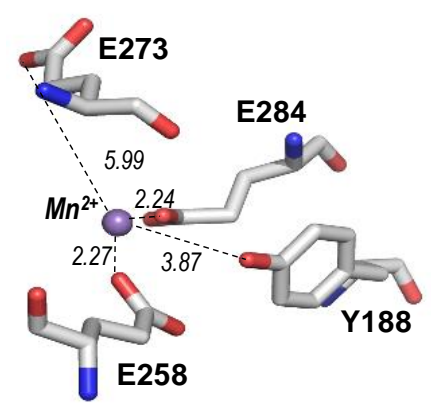

AspDyP2

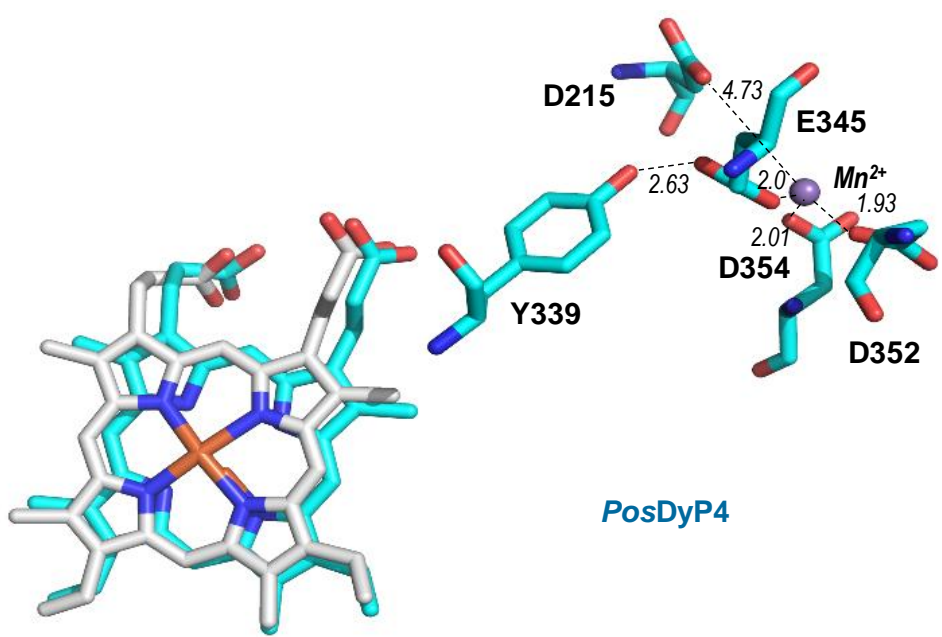

Figure 8. Superposition of the heme cofactors showing the mirror position of the $\mathrm{Mn}^{2+}$ oxidation sites in the DyPs of P. ostreatus (after ligand diffusion with PELE on PDB: 6fsk) and Amycolatopsis sp (as found in PDB: 4g2c). Residues are shown as CPK-colored sticks with blue and white carbons, respectively, and $\mathrm{Mn}^{2+}$ ions as spheres. Residue numbering in AspDyP2 is based on Brown et al. ${ }^{21}$ Some distances are indicated in angstroms.

Up to this study, two $\mathrm{Mn}^{2+}$-binding sites have been reported in DyPs from Rhodococcus jostii RHA1, ${ }^{26}$ and its N246A variant with improved $\mathrm{Mn}^{2+}$-oxidation activity, ${ }^{23}$ and from Amycolatopsis sp 75iv2. ${ }^{21}$ Although crystallographic evidence was provided in these cases, including Mn-anomalous map of the $R$. jostii variant, no experimental confirmation on their involvement in the $\mathrm{Mn}^{2+}$ oxidation reaction has been reported. Even though the location of the manganese-binding site in $R$. jostii DyPB is reminiscent (but at $4 \AA$ distance from heme) of the one described for class II peroxidases, metal coordnation is limited to only water molecules (PDB: 4HOV) hindering its fixation and electron transfer. In contrast, the $\mathrm{Mn}^{2+}$ binding site in the Amycolatopsis sp DyP2, which has much better Mn-oxidation activity than $R$. jostii DyP ${ }^{21}$ is analogous to the one characterized here in PosDyP4, appearing as mirror images when the heme cofactors are superimposed (Figure 8). In both cases, the manganese is located at the protein surface (Figure S10), far from the heme group, and a direct oxidation is not possible. However, this limitation is solved by transferring the electron via an intermediate tyrosine residue, as demonstrated here for PosDyP4. Interestingly, the more distant residue in the PosDyP4 $\mathrm{Mn}^{2+}$-binding site (Asp215) is equivalent to $A$ spDyP2 Glu273, which was reported by Brown et al. ${ }^{21}$ to occupy a second position in the coordination sphere of the $\mathrm{Mn}^{2+}$ ion. The latter authors also reported that the four amino acids involved in $\mathrm{Mn}^{2+}$ oxidation (one tyrosine and three acidic residues) are conserved in a few other DyP-type prokaryotic sequences, in addition to AspDyP4. ${ }^{21}$

\section{CONCLUSIONS}

As illustrated in the scheme of Figure 9 (upper arrow) we demonstrate for the first time (using directed mutagenesis) that oxidation of $\mathrm{Mn}^{2+}$ by a fungal DyP, from P. ostreatus, and probably also by some prokaryotic DyPs, such as that from Amycolatopsis sp 75iv2, is produced at the protein surface ( $>11 \AA$ from the heme) implying a long-range electron transfer pathway. Four acidic residues (and two water molecules) coordinate the metal ion at this position. One of these residues (Glu345) would receive the electron from $\mathrm{Mn}^{2+}$ transferring it to a neighbor tyrosine (Tyr339), which is absolutely necessary for the oxidation reaction to take place. Among fungal ligninolytic peroxidases, the involvement of a tyrosine residue in electron transfer from reducing substrate to heme has been reported only for the lignin peroxidase of Trametopsis cervina. ${ }^{48}$ In contrast, tyrosine involvement in electron transfer for $\mathrm{Mn}^{2+}$-oxidation, as suggested for the first time by Brown et al. ${ }^{21}$ in AspDyP2, seems to be produced in distant DyPs of both eukaryotic and prokaryotic origins. 


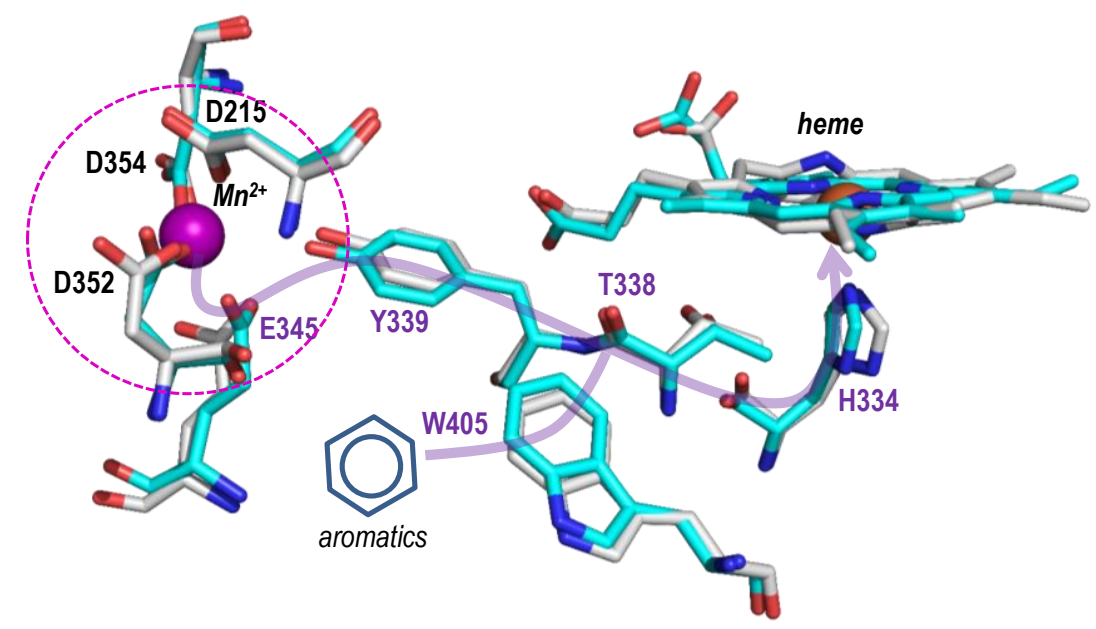

Figure 9. Proposed long-range electron transfer pathways (thick arrows) to the PosDyP4 heme for the oxidation of $\mathrm{Mn}^{2+}$ at the new site described (dashed circle) and oxidation of aromatics at the catalytic Trp405. Displacement of several side chains during $\mathrm{Mn}^{2+}$ binding are shown by superposition of the molecular structures before (CPK colored) and after (cyan colored) PELE simulations.

QM/MM calculations predicted the involvement of Tyr339 in the electron transfer from $\mathrm{Mn}^{2+}$ to the heme, as confirmed later by directed mutagenesis of PosDyP4. EPR spectra are consistent with the formation of a tyrosyl radical in the $\mathrm{H}_{2} \mathrm{O}_{2}$-activated $\mathrm{W} 405 \mathrm{~S}$ variant oxidizing $\mathrm{Mn}^{2+}$, which in the native enzyme overlapped with a tryptophanyl radical. The latter would correspond to the solvent-exposed Trp405 radical involved in oxidation of aromatic substrates. Its formation was also predicted by QM/MM and confirmed by the EPR spectrum of the W405S variant, where only the tyrosyl radical component was observed. The abstracted electron must travel from the exposed Trp405 to the heme, the final part of the route being shared with the $\mathrm{Mn}^{2+}$ oxidation pathway described above (Figure 9, lower arrow).

The new $\mathrm{Mn}^{2+}$ oxidation site, whose operation is demonstrated, could contribute to microbial biodegradation of lignin, acting on its phenolic moiety via $\mathrm{Mn}^{3+}$ chelates, as well as in the subsequent transformation of lignin-derived products. The $\mathrm{Mn}^{3+}$ chelates could also act on the non-phenolic lignin by promoting the formation of reactive lipid radicals, as proposed for selective degradation of lignin by some white-rot fungi. ${ }^{50}$ DyPs could have a relevant contribution to the above reactions, especially in the case of the bacterial DyPs oxidizing $\mathrm{Mn}^{2+}$, such as those from Amycolatopsis species, since no typical ligninolytic peroxidases have been described in prokaryotes, despite the likely bacterial contribution to degradation of lignin products and their incorporation to soil humus. ${ }^{51}$

\section{aUTHOR INFORMATION}

\section{Corresponding Author}

*E-mail: ATMartínez@cib.csic.es

\section{ORCID}

AT Martínez: https://orcid.org/0000-0002-1584$\underline{2863}$

\section{Notes}

The authors declare no competing financial interest.

${ }^{\ddagger}$ Current address: Technical University of Delft, Delft, The Netherlands

\section{ASSOCIATED CONTENT}

\section{Supporting Information}

The Supporting Information is available free of charge on the ACS Publications website at DOI $\mathrm{xxx}$.

Crystallographic data (Table S1); PCR primers (Table S2); ABTS oxidation kinetic parameters (Table S3); SDS-PAGE of PosDyP4 (Figure S1); UV-visible spectra of PosDyP4 and seven variants (Figure S2); detail of the PosDyP4 crystal electron-density map (Figure S3); overall crystal structures of PosDyP4 and eleven related proteins (Figure S4); heme access channel in PosDyP4 (Figure S5); comparison of empty $\mathrm{Mn}^{2+}$ binding site in the PosDyP4 crystal structure and after ion binding 
simulation (Figure S6); EPR spectra before and after activation by $\mathrm{H}_{2} \mathrm{O}_{2}$ (Figure S7); Intensity comparison of PosDyP4 and W405S EPR signals (Figure S8); kinetics of $\mathrm{Mn}^{2+}$ oxidation in non-logarithmic scale (Figure S9); and solvent access surface of $P$. ostreatus and Amycolatopsis sp DyPs showing $\mathrm{Mn}^{2+}$ binding (Figure S10) (PDF file).

\section{ACKNOWLEDGEMENTS}

This work has been funded by the H2020 BBIJU (www.bbi-europe.eu) project EnzOx2 (BBIPPP-2015-2-720297; www.enzox2.eu) and the FP7 project INDOX (KBBE-2013-7-613549; www.indoxproject.eu), together with the BIO2014-56388-R (NOESIS), BFU201677835-R, CTQ2016-79138-R and (BIO201786559-R (GENOBIOREF) projects of the Spanish Ministry of Economy, Industry and Competitiveness, co-financed by FEDER funds. The sequencing of $P$. ostreatus genome at JGI was supported by the DOE Office of Science, under Contract No. DE-AC02-05CH11231. We thank the staff of the ID30B beamline at ESRF (Grenoble, France) for assistance. CSGI (Florence, Italy) FFABR 2017 (Legge 232/2016 Comma 295, and Legge di Bilancio 2017) and MIUR (Dipartimento di Eccellenza, 2018-2022) grants are acknowledged for financial support.

\section{- REFERENCES}

(1) Welinder,K.G., Superfamily of plant, fungal and bacterial peroxidases. Curr. Opin. Struct. Biol. 1992, 2, 388-393.

(2) Hofrichter,M.; Ullrich,R.; Pecyna,M.J.; Liers,C.; Lundell,T., New and classic families of secreted fungal heme peroxidases. Appl. Microbiol. Biotechnol. 2010, 87, 871-897.

(3) Rahmanpour,R.; Bugg,T.D., Structure and reactivity of the dye-decolorizing peroxidase (DyP) family; In Heme peroxidases; Raven,E.L.; Dunford,H.B., Eds.; RSC: London, 2015, pp 334357.

(4) Sugano,Y., DyP-type peroxidases comprise a novel heme peroxidase family. Cell Mol. Life Sci. 2009, 66, 1387-1403.

(5) Linde,D.; Ruiz-Dueñas,F.J.; FernándezFueyo,E.; Guallar,V.; Hammel,K.E.; Pogni,R.; Martínez,A.T., Basidiomycete DyPs: Genomic diversity, structural-functional aspects, reaction mechanism and environmental significance. Arch. Biochem. Biophys. 2015, 574, 66-74.
(6) Goblirsch,B.; Kurker,R.C.; Streit,B.R.; Wilmot,C.M.; Dubois,J.L., Chlorite dismutases, DyPs, and EfeB: 3 microbial heme enzyme families comprise the CDE structural superfamily. J. Mol. Biol. 2011, 408, 379-398.

(7) Kim,S.J.; Shoda,M., Purification and characterization of a novel peroxidase from Geotrichum candidum Dec 1 involved in decolorization of dyes. Appl. Environ. Microbiol. 1999, 65, 1029-1035.

(8) Yoshida,T.; Tsuge,H.; Konno,H.; Hisabori,T.; Sugano,Y., The catalytic mechanism of dye-decolorizing peroxidase DyP may require the swinging movement of an aspartic acid residue. FEBS J. 2011, 278, 2387-2394.

(9) Strittmatter,E.; Liers,C.; Ullrich,R.; Wachter,S.; Hofrichter,M.; Plattner,D.A.; Piontek,K., First crystal structure of a fungal highredox potential dye-decolorizing peroxidase: Substrate interaction sites and long-range electron transfer. J. Biol. Chem. 2013, 288, 4095-4102.

(10) Ruiz-Dueñas,F.J.; Morales,M.; García,E.; Miki,Y.; Martínez,M.J.; Martínez,A.T., Substrate oxidation sites in versatile peroxidase and other basidiomycete peroxidases. J. Exp. Bot. 2009, 60, 441-452.

(11) Sugano,Y.; Muramatsu,R.; Ichiyanagi,A.; Sato,T.; Shoda,M., DyP, a unique dye-decolorizing peroxidase, represents a novel heme peroxidase family. Asp171 replaces the distal histidine of classical peroxidases. J. Biol. Chem. 2007, 282, 36652-36658.

(12) Singh,R.; Eltis,L.D., The multihued palette of dye-decolorizing peroxidases. Arch. Biochem. Biophys. 2015, 574, 56-65.

(13) Linde,D.; Coscolín,C.; Liers,C.; Hofrichter,M.; Martínez,A.T.; Ruiz-Dueñas,F.J., Heterologous expression and physicochemical characterization of a fungal dye-decolorizing peroxidase from Auricularia auricula-judae. Protein Express. Purif. 2014, 103, 28-37.

(14) Behrens,C.J.; Zelena,K.; Berger,R.G., Comparative cold shock expression and characterization of fungal dye-decolorizing peroxidases. Appl. Biochem. Biotechnol. 2016, 179, 1404-1417.

(15) Fernández-Fueyo,E.; Linde,D.; Almendral,D.; López-Lucendo,M.F.; RuizDueñas,F.J.; Martínez,A.T., Description of the first fungal dye-decolorizing peroxidase oxidizing manganese(II). Appl. Microbiol. Biotechnol. 2015, 99, 8927-8942.

(16) Chen,C.; Shrestha,R.; Jia,K.; Gao,P.F.; Geisbrecht,B.V.; Bossmann,S.H.; Shi,J.; Li,P., Characterization of dye-decolorizing peroxidase (DyP) from Thermomonospora curvata reveals 
unique catalytic properties of A-type DyPs. J. Biol. Chem. 2015, 290, 23447-23463.

(17) Mendes,S.; Brissos,V.; Gabriel,A.; Catarino,T.; Turner,D.L.; Todorovic,S.; Martins,L.O., An integrated view of redox and catalytic properties of B-type PpDyP from Pseudomonas putida MET94 and its distal variants. Arch. Biochem. Biophys. 2015, 574, 99-107.

(18) Shrestha,R.; Chen,X.; Ramyar,K.X.; Hayati,Z.; Carlson,E.A.; Bossmann,S.H.; Song,L.; Geisbrecht,B.V.; Li,P., Identification of surfaceexposed protein radicals and a substrate oxidation site in A-class dye-decolorizing peroxidase from Thermomonospora curvata. ACS Catal. 2016, 6, 8036-8047.

(19) Shrestha,R.; Huang,G.; Meekins,D.A.; Geisbrecht,B.V.; Li,P., Mechanistic insights into dye-decolorizing peroxidase revealed by solvent isotope and viscosity effects. ACS Catal. 2017, 7, 6352-6364.

(20) Loncar,N.; Colpa,D.I.; Fraaije,M.W., Exploring the biocatalytic potential of a DyP-type peroxidase by profiling the substrate acceptance of Thermobifida fusca DyP peroxidase. Tetrahedron 2016, 72, 7276-7281.

(21) Brown,M.E.; Barros,T.; Chang,M.C.Y., Identification and characterization of a multifunctional dye peroxidase from a ligninreactive bacterium. ACS Chem. Biol. 2012, 7, 20742081.

(22) Duan,Z.; Shen,R.; Liu,B.; Yao,M.; Jia,R., Comprehensive investigation of a dye-decolorizing peroxidase and a manganese peroxidase from Irpex lacteus $\mathrm{F} 17$, a lignin-degrading basidiomycete. $A M B$ Express 2018, 8, 119.

(23) Singh,R.; Grigg,J.C.; Qin,W.; Kadla,J.F.; Murphy,M.E.P.; Eltis,L.D., Improved manganeseoxidizing activity of DypB, a peroxidase from a lignolytic bacterium. ACS Chem. Biol. 2013, 8, 700706.

(24) Santos,A.; Mendes,S.; Brissos,V.; Martins,L.O., New dye-decolorizing peroxidases from Bacillus subtilis and Pseudomonas putida MET94: towards biotechnological applications. Appl. Microbiol. Biotechnol. 2014, 98, 2053-2065.

(25) Rahmanpour,R.; Bugg,T.D.,

Characterisation of DyP-type peroxidases from Pseudomonas fluorescens Pf-5: Oxidation of $\mathrm{Mn}$ (II) and polymeric lignin by Dyp1B. Arch. Biochem. Biophys. 2015, 574, 93-98.

(26) Roberts,J.N.; Singh,R.; Grigg,J.C.; Murphy,M.E.P.; Bugg,T.D.H.; Eltis,L.D., Characterization of dye-decolorizing peroxidases from Rhodococcus jostii RHA1. Biochemistry 2011, 50, 5108-5119.

(27) Kolwek,J.; Behrens,C.; Linke,D.; Krings,U.; Berger,R.G., Cell-free one-pot conversion of (+)- valencene to (+)-nootkatone by a unique dyedecolorizing peroxidase combined with a laccase from Funalia trogii. J. Ind. Microbiol. Biotechnol. 2018, 45, 89-101.

(28) Kellner,H.; Luis,P.; Pecyna,M.J.; Barbi,F.; Kapturska,D.; Krüger,D.; Zak,D.R.; Marmeisse,R.; Vandenbol,M.; Hofrichter,M., Widespread occurrence of expressed fungal secretory peroxidases in forest soils. PLoS ONE 2014, 9: e95557.

(29) Ahmad,M.; Roberts,J.N.; Hardiman,E.M.; Singh,R.; Eltis,L.D.; Bugg,T.D.H., Identification of DypB from Rhodococcus jostii RHA1 as a lignin peroxidase. Biochemistry 2011, 50, 5096-5107.

(30) Martínez,A.T.; Camarero,S.; Ruiz-

Dueñas,F.J.; Martínez,M.J., Biological lignin degradation; In Lignin valorization: Emerging approaches; Beckham,G.T., Ed.; Royal Society of Chemistry: London, 2018, pp 199-225.

(31) Brissos,V.; Tavares,D.; Sousa,A.C.; Robalo,M.P.; Martins,L.O., Engineering a bacterial DyP-type peroxidase for enhanced oxidation of lignin-related phenolics at alkaline $\mathrm{pH}$. ACS Catal. 2017, 7, 3454-3465.

(32) Rahmanpour,R.; Rea,D.; Jamshidi,S.; Fueloep,V.; Bugg,T.D., Structure of Thermobifida fusca DyP-type peroxidase and activity towards Kraft lignin and lignin model compounds. Arch. Biochem. Biophys. 2016, 594, 54-60.

(33) Studier,F.W., Protein production by autoinduction in high-density shaking cultures. Protein Express. Purif. 2005, 41, 207-234.

(34) Kabsch,W., XDS. Acta Crystallogr. D. Biol. Crystallogr. 2010, 66, 125-132.

(35) Collaborative Computational Project,N.4., The CCP4 suite: Programs for protein crystallography. Acta Crystallogr. D. Biol. Crystallogr. 1994, 50, 760-763.

(36) Emsley,P.; Cowtan,K., Coot: modelbuilding tools for molecular graphics. Acta Crystallogr. D. Biol. Crystallogr. 2004, 60, 2132.

(37) Chen,V.B.; Arendall,W.B.; Headd,J.J.; Keedy,D.A.; Immormino,R.M.; Kapral,G.J.; Murray,L.W.; Richardson,J.S.; Richardson,D.C., MolProbity: all-atom structure validation for macromolecular crystallography. Acta Crystallogr.

D. Biol. Crystallogr. 2010, 66, 12-21.

(38) Schrödinger Inc; QSite 4.5; Schrödinger, Inc.: Portland, Oregon, 2007.

(39) Acebes,S.; Fernández-Fueyo,E.; Monza,E.; Lucas,F.; Almendral,D.; Ruiz-Dueñas,F.J.; Lund,H.; Martínez,A.T.; Guallar,V., Rational enzyme engineering through biophysical and biochemical modeling. ACS Catal. 2016, 6, 1624-1629.

(40) Lecina,D.; Gilabert,J.F.; Guallar,V., Adaptive simulations, towards interactive proteinligand modeling. Sci. Rep. 2017, 7, 8466. 
(41) Guallar,V.; Wallrapp,F.H., QM/MM methods: Looking inside heme proteins biochemisty. Biophys. Chem. 2010, 149, 1-11.

(42) Guallar,V.; Wallrapp,F., Mapping protein electron transfer pathways with QM/MM methods. J. R. Soc. Interface 2008, 5, S233-S239.

(43) Linde,D.; Pogni,R.; Cañellas,M.; Lucas,F.; Guallar,V.; Baratto,M.C.; Sinicropi,A.; SáezJiménez,V.; Coscolín,C.; Romero,A.; Medrano,F.J.; Ruiz-Dueñas,F.J.; Martínez,A.T., Catalytic surface radical in dye-decolorizing peroxidase: A computational, spectroscopic and directed mutagenesis study. Biochem. J. 2015, 466, 253-262.

(44) Holm,L.; Rosenstrom,P., Dali server: conservation mapping in 3D. Nucleic Acids Res. 2010, 38, W545-W549.

(45) Yoshida,T.; Sugano,Y., A structural and functional perspective of DyP-type peroxidase family. Arch. Biochem. Biophys. 2015, 574, 49-55.

(46) Uchida,T.; Sasaki,M.; Tanaka,Y.; Ishimorit,K., A dye-decolorizing peroxidase from Vibrio cholerae. Biochemistry 2015, 54, 6610-6621.

(47) Baratto,M.C.; Sinicropi,A.; Linde,D.; SáezJiménez,V.; Sorace,L.; Ruiz-Dueñas,F.J.; Martínez,A.T.; Basosi,R.; Pogni,R., Redox-active sites in Auricularia auricula-judae dye-decolorizing peroxidase and several directed variants: A multifrequency EPR study. J. Phys. Chem. B 2015, 119, 13583-13592.

(48) Miki,Y.; Calviño,F.R.; Pogni,R.; Giansanti,S.; Ruiz-Dueñas,F.J.; Martínez,M.J.; Basosi,R.; Romero,A.; Martínez,A.T., Crystallographic, kinetic, and spectroscopic study of the first ligninolytic peroxidase presenting a catalytic tyrosine. J. Biol. Chem. 2011, 286, 1552515534.

(49) Gold,M.H.; Youngs,H.L.; Gelpke,M.D., Manganese peroxidase. Met. Ions Biol. Syst. 2000, 37, 559-586.

(50) Fernández-Fueyo,E.; Ruiz-Dueñas,F.J.; Ferreira,P.; Floudas,D.; Hibbett,D.S.; Canessa,P.; Larrondo,L.; James,T.Y.; Seelenfreund,D.; Lobos,S.; Polanco,R.; Tello,M.; Honda,Y.; Watanabe,T.; Watanabe,T.; Ryu,J.S.; Kubicek,C.P.; Schmoll,M.; Gaskell,J.; Hammel,K.E.; St.John,F.J.; Vanden Wymelenberg,A.; Sabat,G.; Bondurant,S.S.; Syed,K.; Yadav,J.; Doddapaneni,H.;

Subramanian,V.; Lavín,J.L.; Oguiza,J.A.; Perez,G.; Pisabarro,A.G.; Ramírez,L.; Santoyo,F.; Master,E.; Coutinho,P.M.; Henrissat,B.; Lombard,V.; Magnuson,J.K.; Kües,U.; Hori,C.; Igarashi,K.; Samejima,M.; Held,B.W.; Barry,K.; LaButti,K.; Lapidus,A.; Lindquist,E.; Lucas,S.; Riley,R.; Salamov,A.; Hoffmeister,D.; Schwenk,D.; Hadar,Y.; Yarden,O.; de Vries,R.P.; Wiebenga,A.; Stenlid,J.; Eastwood,D.C.; Grigoriev,I.V.; Berka,R.;

Blanchette,R.A.; Kersten,P.; Martínez,A.T.; Vicuña,R.; Cullen,D., Comparative genomics of Ceriporiopisis subvermispora and Phanerochaete chrysosporium provide insight into selective ligninolysis. Proc. Natl. Acad. Sci. USA 2012, 109, 5458-5463.

(51) de Gonzalo,G.; Colpa,D.I.; Habib,M.H.M.; Fraaije,M.W., Bacterial enzymes involved in lignin degradation. J. Biotechnol. 2016, 236, 110-119. 
TOC Image:

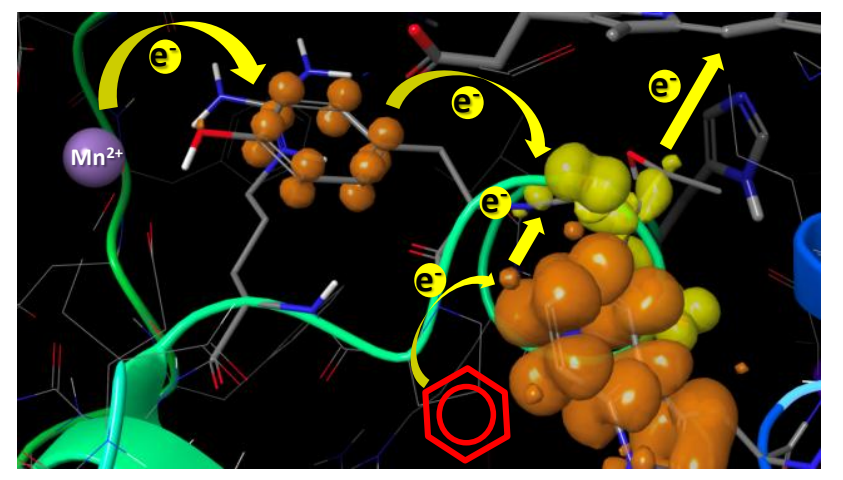




\section{SUPPORTING INFORMATION}

\section{Description of a Non-Canonical Mn(II)-Oxidation Site in Peroxidases}

Elena Fernández-Fueyo, ${ }^{\S}$ Irene Davó-Siguero, ${ }^{\S}$ David Almendral, ${ }^{\S}$ Dolores Linde, ${ }^{\S}$ Maria Camilla Baratto, ${ }^{\#}$ Rebecca Pogni, ${ }^{\#}$ Antonio Romero, ${ }^{\S}$ Victor Guallar, ${ }^{\text {I† }}$ and Angel T. Martínez*\$

${ }^{\S}$ Centro de Investigaciones Biológicas, CSIC, Ramiro de Maeztu 9, E-28006 Madrid, Spain; "Department of Biotechnology, Chemistry and Pharmacy, University of Siena, I-53100 Siena, Italy; ${ }_{\text {Marcelona }}$ Supercomputing Center, Jordi Girona 29, E-08034 Barcelona, Spain; `ICREA, Passeig Lluís Companys 23, E-08010 Barcelona, Spain

*E-mail: ATMartinez@cib.csic.es

This Supporting Information includes crystallographic data (Table S1); PCR primers (Table S2); ABTS oxidation kinetic parameters (Table S3); SDS-PAGE of PosDyP4 (Figure S1); UV-visible spectra of PosDyP4 and seven variants (Figure S2); detail of the PosDyP4 crystal electron-density map (Figure S3); overall crystal structures of PosDyP4 and eleven related proteins (Figure S4); heme access channel in PosDyP4 (Figure S5); comparison of empty $\mathrm{Mn}^{2+}$ binding site in the PosDyP4 crystal structure and after ion binding simulation (Figure S6); EPR spectra before and after activation by $\mathrm{H}_{2} \mathrm{O}_{2}$ (Figure S7); Intensity comparison of PosDyP4 and W405S EPR signals (Figure S8); kinetics of $\mathrm{Mn}^{2+}$ oxidation in non-logarithmic scale (Figure S9); and solvent access surface of P. ostreatus and Amycolatopsis sp DyPs showing $\mathrm{Mn}^{2+}$ binding (Figure S10) (PDF file). 
Table S1. X-ray data collection and refinement statistics for PosDyP4 (data in parenthesis correspond to the last resolution layer).

\begin{tabular}{|c|c|}
\hline \multicolumn{2}{|l|}{ Data collection: } \\
\hline Wavelength $(\AA ̊)$ & 0.9762 \\
\hline Crystal system, space group & Orthorhombic, $\mathrm{P} 22_{1} 2_{1}$ \\
\hline Unit cell parameters $(\AA)$ & $a=62.72, b=117.57, c=140.43$ \\
\hline Resolution range $(\AA)^{*}$ & $70.22-1.56(1.64-1.56)$ \\
\hline $\mathrm{N}^{0}$ of observations & $655645(96513)$ \\
\hline $\mathrm{N}^{0}$ of unique reflections & 144926 (20996) \\
\hline Redundancy & $4.5(4.6)$ \\
\hline Completeness (\%) & $97.3(97.4)$ \\
\hline Mean $I \sigma(I)$ & $12.7(2.3)$ \\
\hline$R_{\text {merge }}{ }^{a}$ & $0.065(0.514)$ \\
\hline $\mathrm{R}_{\text {meas }} \mathrm{b}$ & $0.083(0.649)$ \\
\hline $\mathrm{CC}_{1 / 2}^{\mathrm{c}}(\%)$ & $99.7(74.5)$ \\
\hline Wilson B-factor $\left(\AA^{2}\right)$ & 17.70 \\
\hline \multicolumn{2}{|l|}{ Refinement: } \\
\hline Resolution range $(\AA)$ & $70.22-1.56(1.60-1.56)$ \\
\hline $\mathrm{N}^{0}$ of working reflections & $137552(10100)$ \\
\hline $\mathrm{N}^{0}$ of testing reflections & $7250(553)$ \\
\hline Completeness (\%) & 97.05 \\
\hline $\mathrm{R}_{\text {work }}$ & $0.162(0.307)$ \\
\hline $\mathrm{R}_{\text {free }} \#$ & $0.199(0.334)$ \\
\hline \multicolumn{2}{|l|}{$N^{0}$ of non-H atoms: } \\
\hline Protein & 7484 \\
\hline Heme group & 86 \\
\hline MES & 12 \\
\hline Water molecules & 1119 \\
\hline \multicolumn{2}{|l|}{ Average B factors $\left(\AA^{2}\right)$ : } \\
\hline Protein & 18.56 \\
\hline Heme group & 10.72 \\
\hline MES & 22.95 \\
\hline Water molecules & 29.70 \\
\hline \multicolumn{2}{|l|}{ RMS deviations: } \\
\hline Bond lengths $(\AA)$ & 0.021 \\
\hline Bond angles $\left({ }^{\circ}\right)$ & 2.04 \\
\hline \multicolumn{2}{|l|}{ Ramachandran plot statistics: } \\
\hline Favoured (\%) & 97.8 \\
\hline Allowed (\%) & 2.0 \\
\hline Outliers (\%) & 0.2 \\
\hline \multicolumn{2}{|l|}{ PDB: } \\
\hline ID code & 6FSK \\
\hline
\end{tabular}




\begin{tabular}{|c|c|c|}
\hline Mutation & Type & Sequence \\
\hline D196A & $\begin{array}{l}\text { fwd } \\
\text { rev }\end{array}$ & 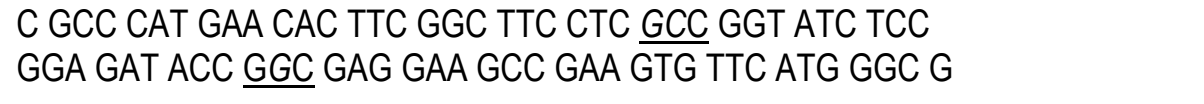 \\
\hline D215V & $\begin{array}{l}\text { fwd } \\
\text { rev }\end{array}$ & $\begin{array}{l}\text { C CCG CTC CCA GGT CAA GTT CCG ATC AGA CCG GGC } \\
\text { GCC CGG TCT GAT CGG } \underline{A \overline{A C} \text { TTG ACC TGG GAG CGG G }}\end{array}$ \\
\hline Y339A & $\begin{array}{l}\text { fwd } \\
\text { rev }\end{array}$ & 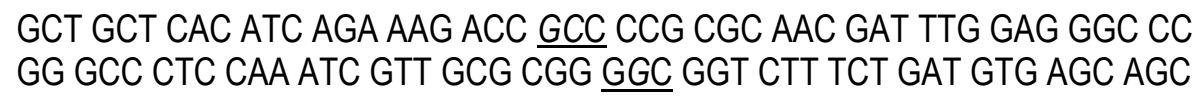 \\
\hline D352A & $\begin{array}{l}\text { fwd } \\
\text { rev } \\
\text { fwd } \\
\text { rev }\end{array}$ & $\begin{array}{l}\text { C CCG CGC AAC GAT TTG GCG GGC CCG CC } \\
\text { GG CGG GCC CGC CAA ATC GTT GCG CGG G } \\
\text { GGC CCG CCT TTG AAA GCT GCT ATT GAC AAC CG } \\
\text { CG GTT GTC AAT AGC AGC TTT CAA AGG CGG GCC }\end{array}$ \\
\hline D354A & $\begin{array}{l}\text { fwd } \\
\text { rev }\end{array}$ & $\begin{array}{l}\text { GG CCC GCC TTTG AAA GCT GAT ATT GCC AAC CGT AGA ATC ATC CGC } \\
\text { GCG GAT GAT TCT ACG GTT GGC AAT ATC AGC TTT CAA AGG CGG GCC }\end{array}$ \\
\hline W405S & $\begin{array}{l}\text { fwd } \\
\text { rev }\end{array}$ & $\begin{array}{l}\text { C CAC TTC ATT CAG GAA TCT TGG GCC AAT GCC C } \\
\text { G GGC ATT GGC CCA AGA TTC CTG AAT GAA GTG G }\end{array}$ \\
\hline D352A/D354A & $\begin{array}{l}\text { fwd } \\
\text { rev }\end{array}$ & $\begin{array}{l}\text { GGC CCG CCT TTG AAA GCT GCT ATT GCC AAC CGT AGA ATC ATC CGC } \\
\text { GCG GAT GAT TCT ACG GTT GGC AAT AGC AGC TTT CAA AGG CGG GCC }\end{array}$ \\
\hline
\end{tabular}

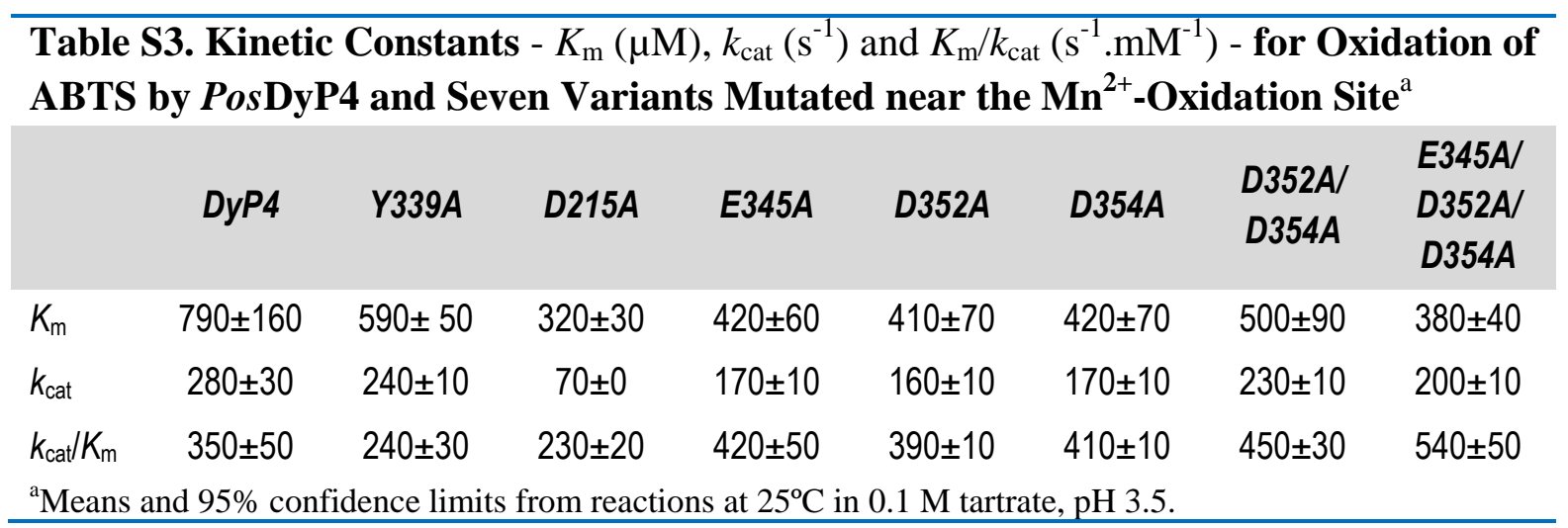




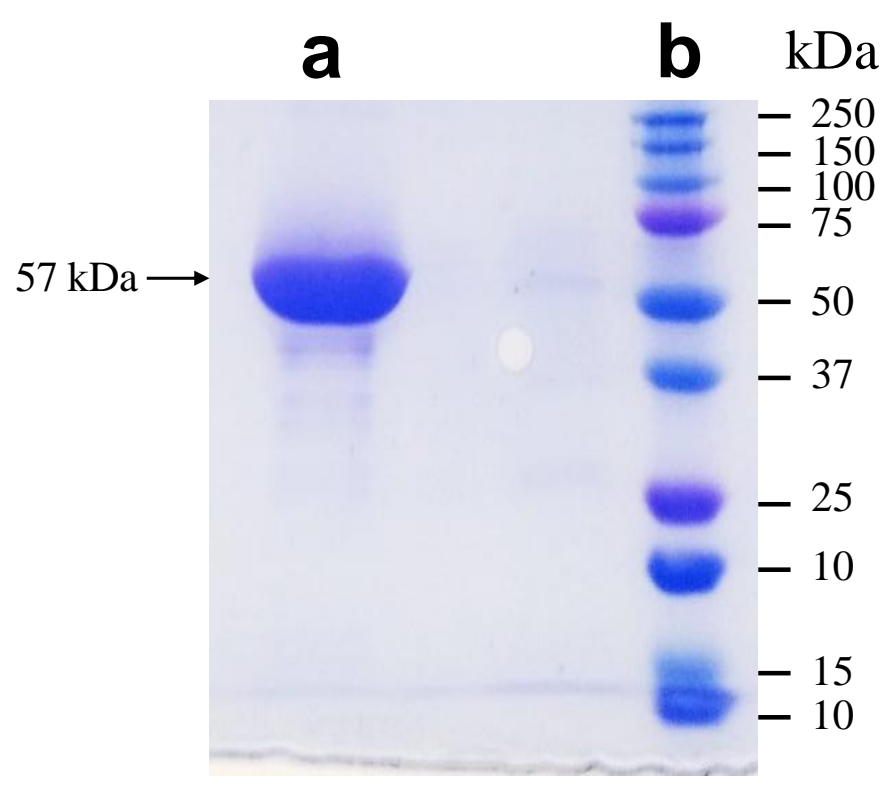

Figure S1. SDS-PAGE of purified PosDyP4 (lane a, arrow) and molecular-mass markers (lane b). 

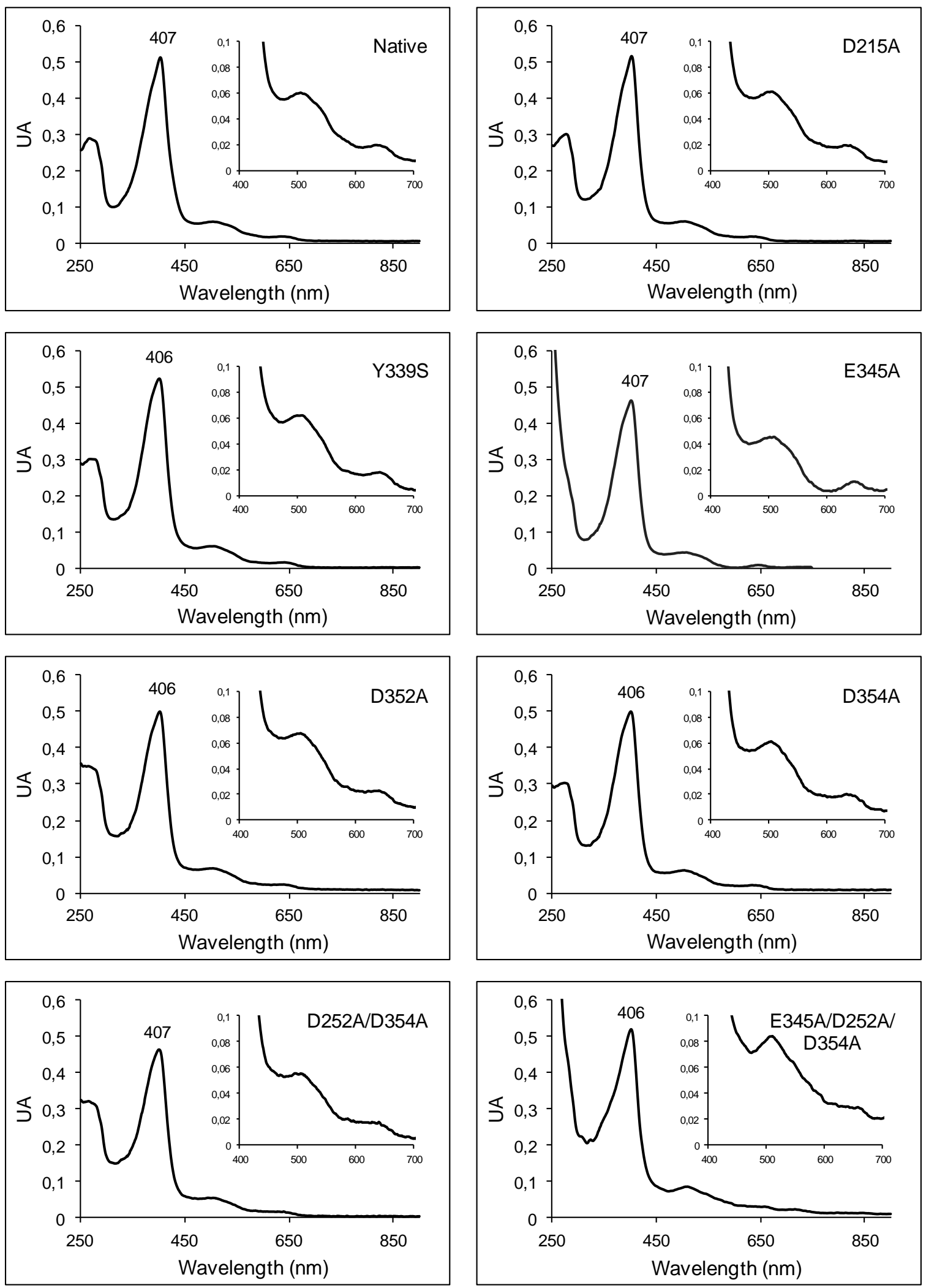

Figure S2. UV-visible spectra of PosDyP4 and seven variants at the $\mathrm{Mn}^{2+}$ oxidation site. The Soret band wavelength and details of the 400-700 nm region are included. 


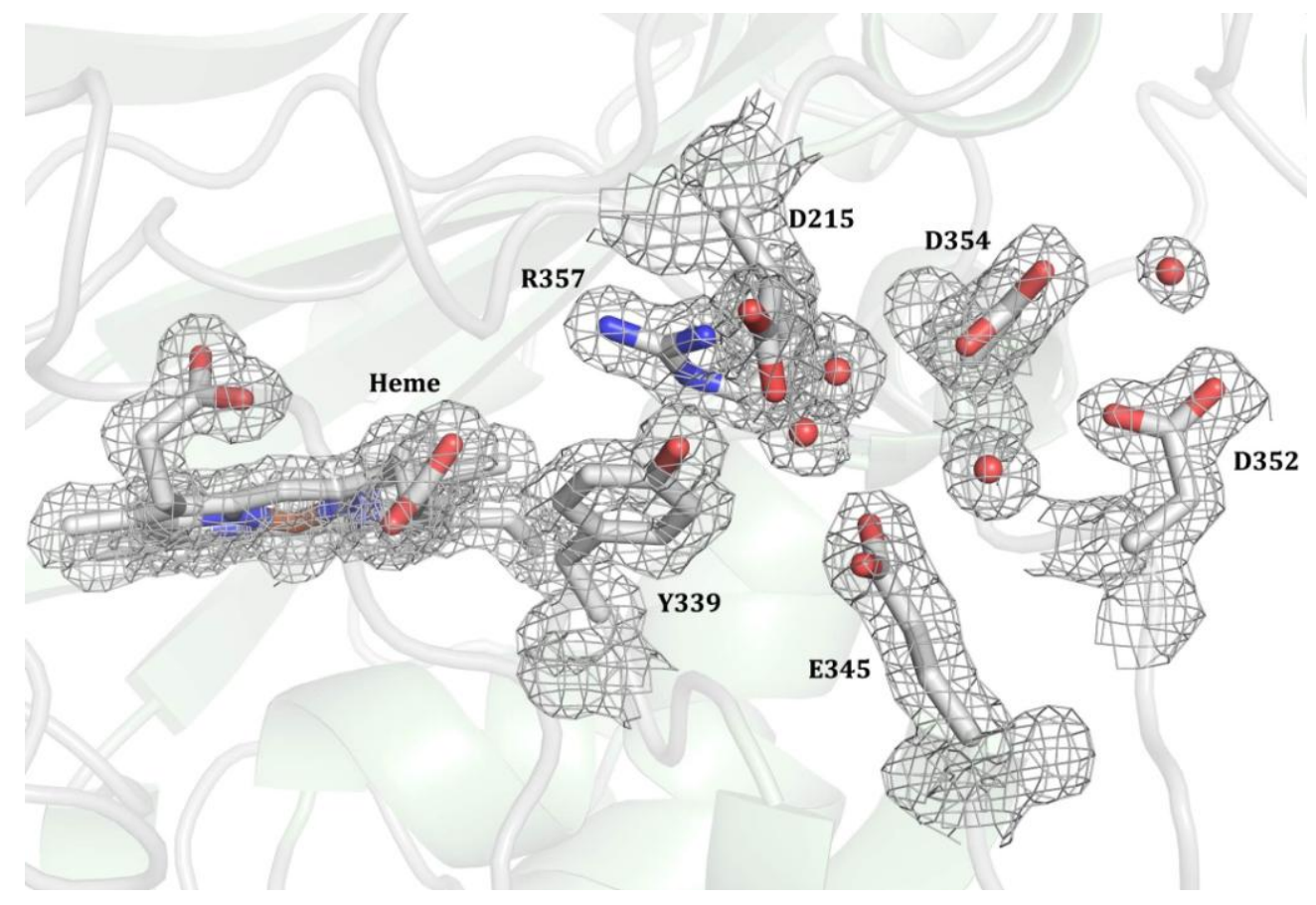

Figure S3. $2 F_{o^{-}} F_{c}$ electron density map, contoured at $1 \sigma$ level, of residues surrounding several water molecules in PosDyP4, and CPK-colored stick representation of these residues in PDB: 6fsk. 

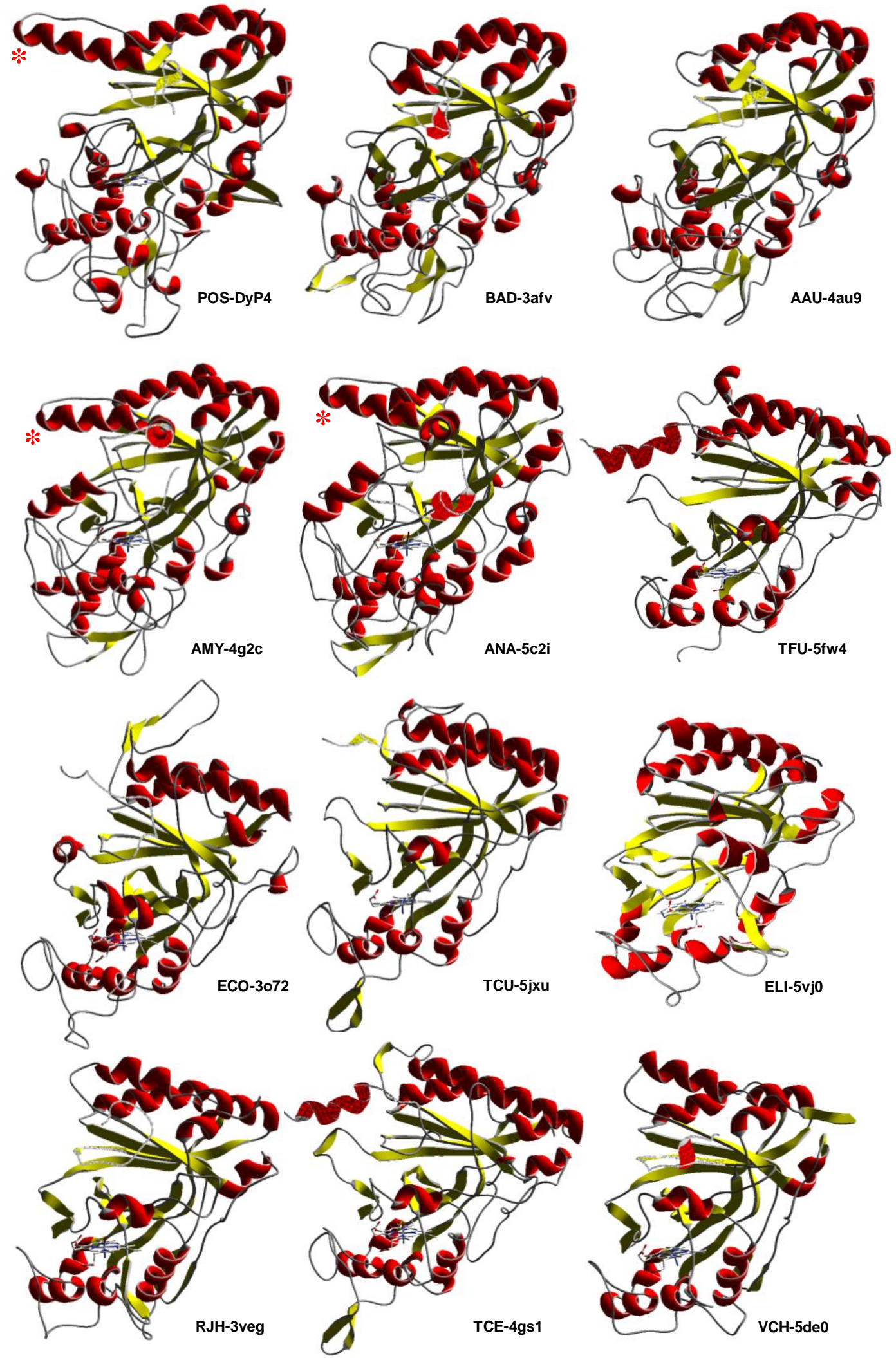

Figure S4. Overall structure of PosDyP4 (6fsk) and selected structurally related proteins of fungal (3afv and 4au9) and prokaryotic origin $(4 \mathrm{~g} 2 \mathrm{c}, 5 \mathrm{c} 2 \mathrm{i}, 5 \mathrm{fw} 4,3 \mathrm{o} 72$, $5 \mathrm{jxu}, 5 \mathrm{vj0}, 3 \mathrm{veg}, 4 \mathrm{gs} 1$ and $5 \mathrm{de} 0$ ). See Table 1 for species names of the different PDB entries (red asterisks indicate prominent helix-8 in three of the structures). 

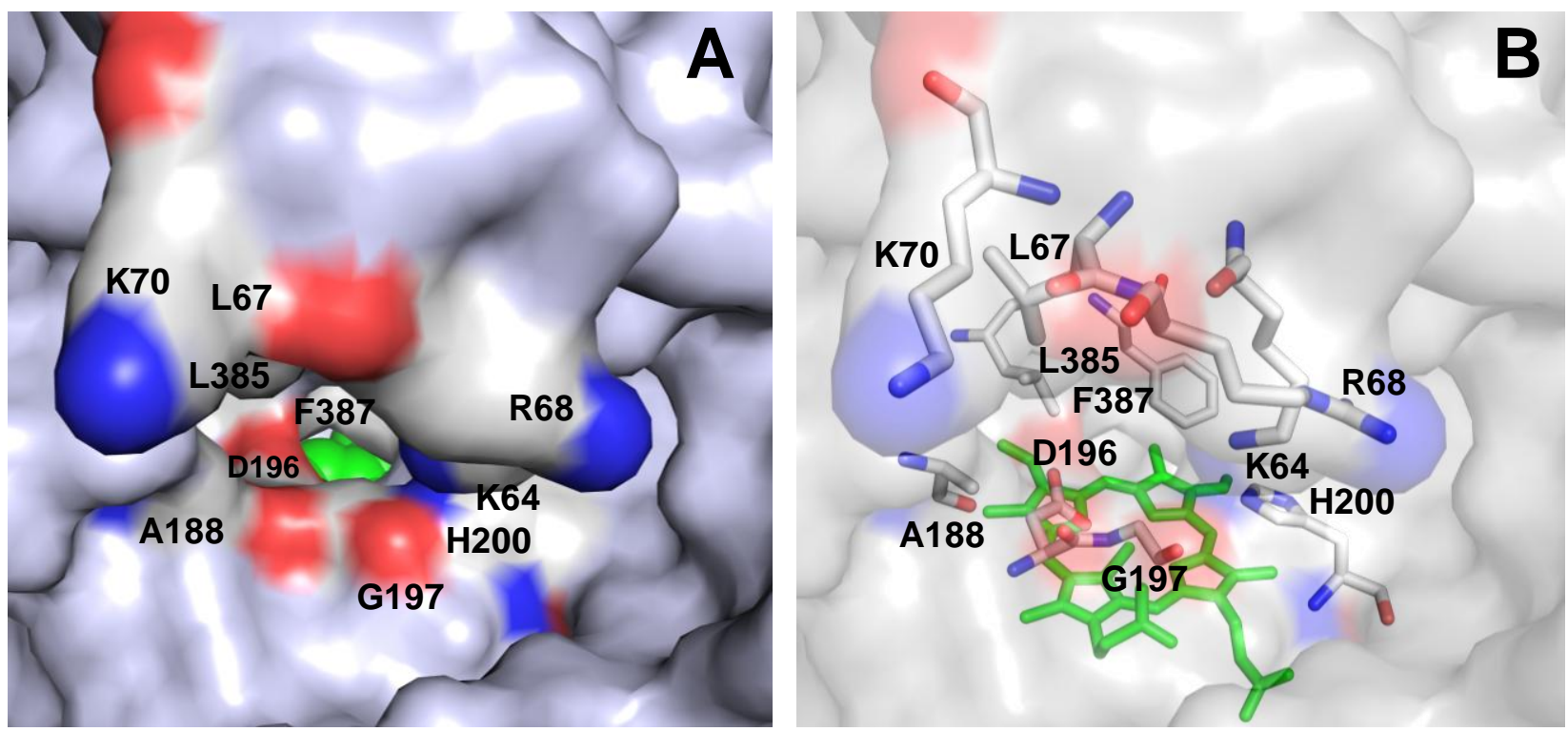

Figure S5. Heme access channel in PosDyP4. A. Detail of solvent access surface with heme (green) and channel residues (CPK colors) as spheres. B. Semitransparent surface showing heme and channel residues as sticks (from PDB: 6fsk). 

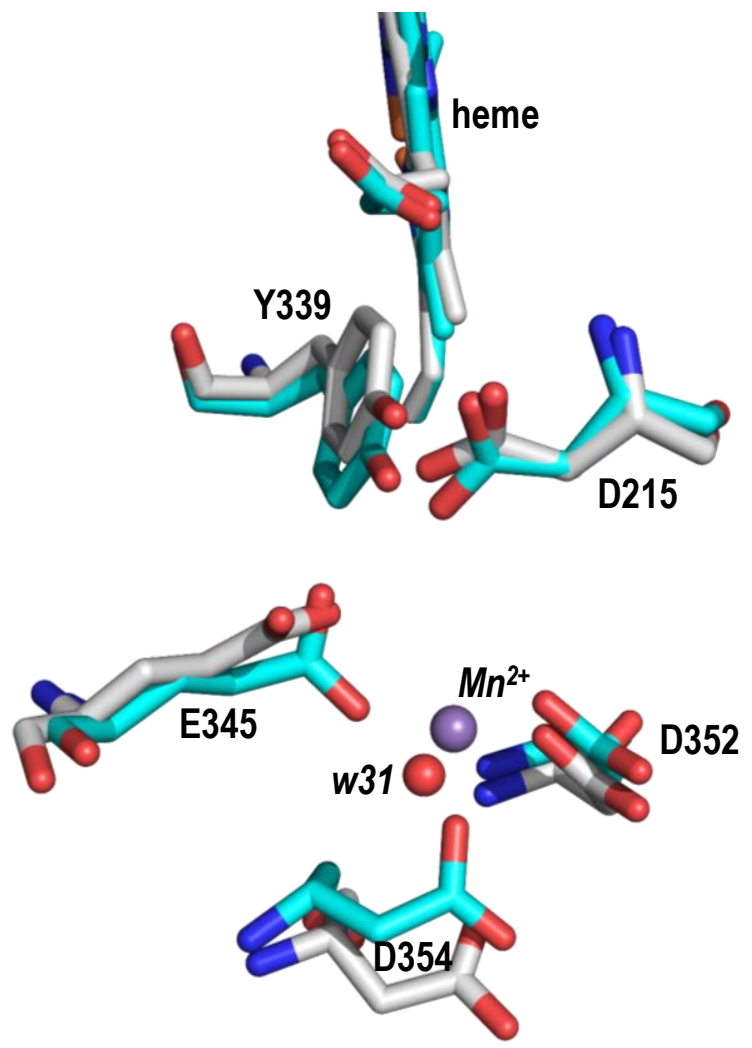

Figure S6. Superimposition of the four acidic residues involved in $\mathrm{Mn}^{2+}$ binding and Tyr339 (Figure 4) before and after ligand diffusion with PELE (as CPKcolored sticks with white and cyan carbons, respectively). The displacement of some side chains for metal ion binding is observed. 


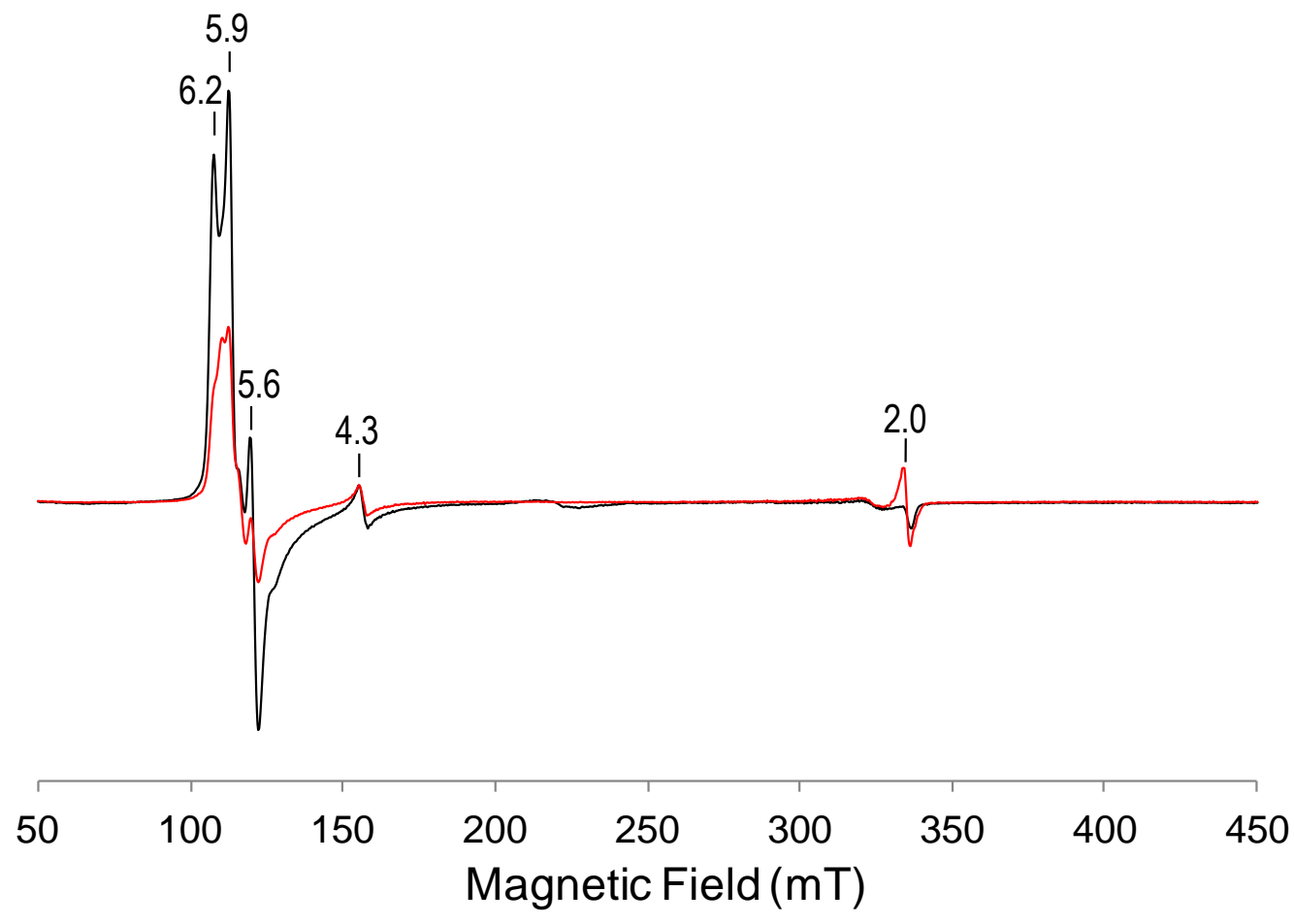

Figure S7. X-band EPR spectra of $0.25 \mathrm{mM}$ native PosDyP4 in tartrate buffer, $\mathrm{pH}$ 3 , before (black line) and after (red line) the addition of 8 eq. of $\mathrm{H}_{2} \mathrm{O}_{2}$. Experimental conditions were $5 \mathrm{~mW}$ microwave power, $0.4 \mathrm{mT}$ modulation amplitude, and $v=9.39 \mathrm{GHz}$. 


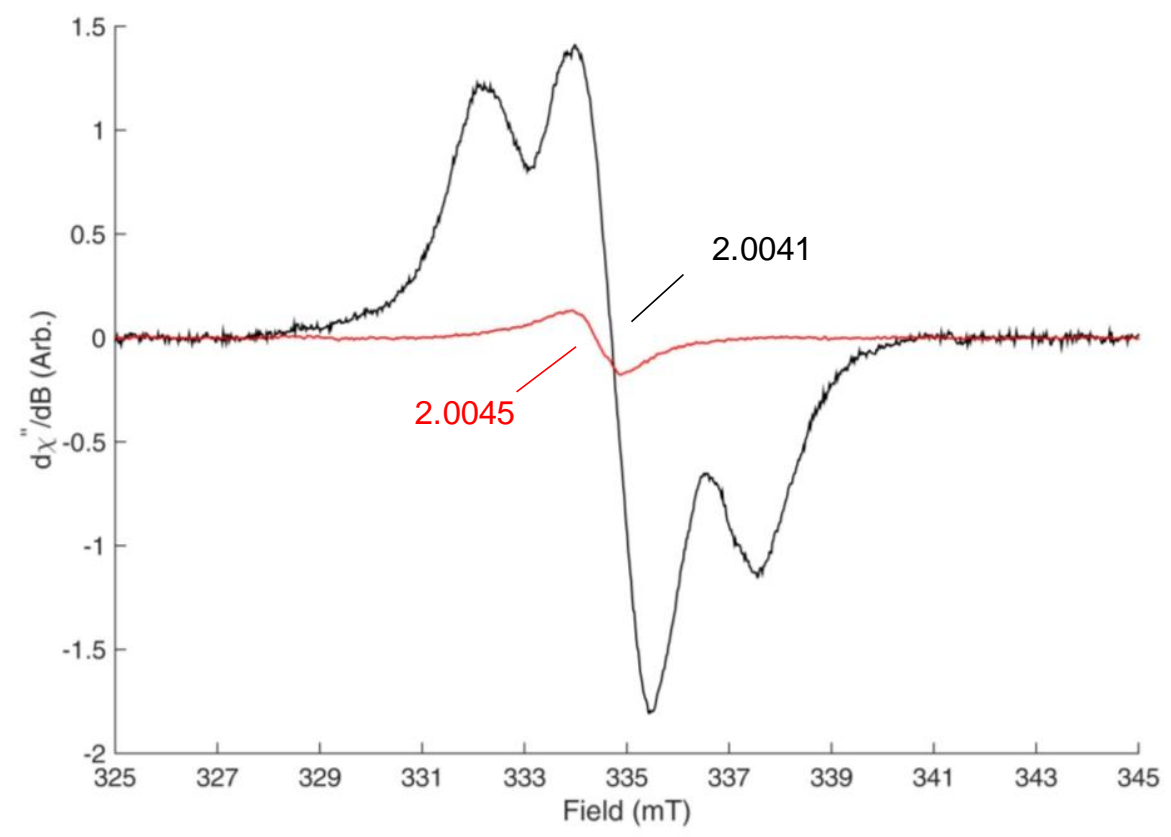

Figure S8. Comparison of $20 \mathrm{mT}$ scan width X-band EPR spectra of $0.25 \mathrm{mM}$ native PosDyP4 (black line) and W405S variant (red line) in tartrate buffer, $\mathrm{pH} 3$, after the addition of 8 eq. of $\mathrm{H}_{2} \mathrm{O}_{2}$. Conditions were $5 \mathrm{~mW}$ microwave power, $0.2 \mathrm{mT}$ modulation amplitude, and $v=9.39$ (PosDyP4) or 9.38 (W405S) GHz. The normalized spectra are shown in Figure 6. 


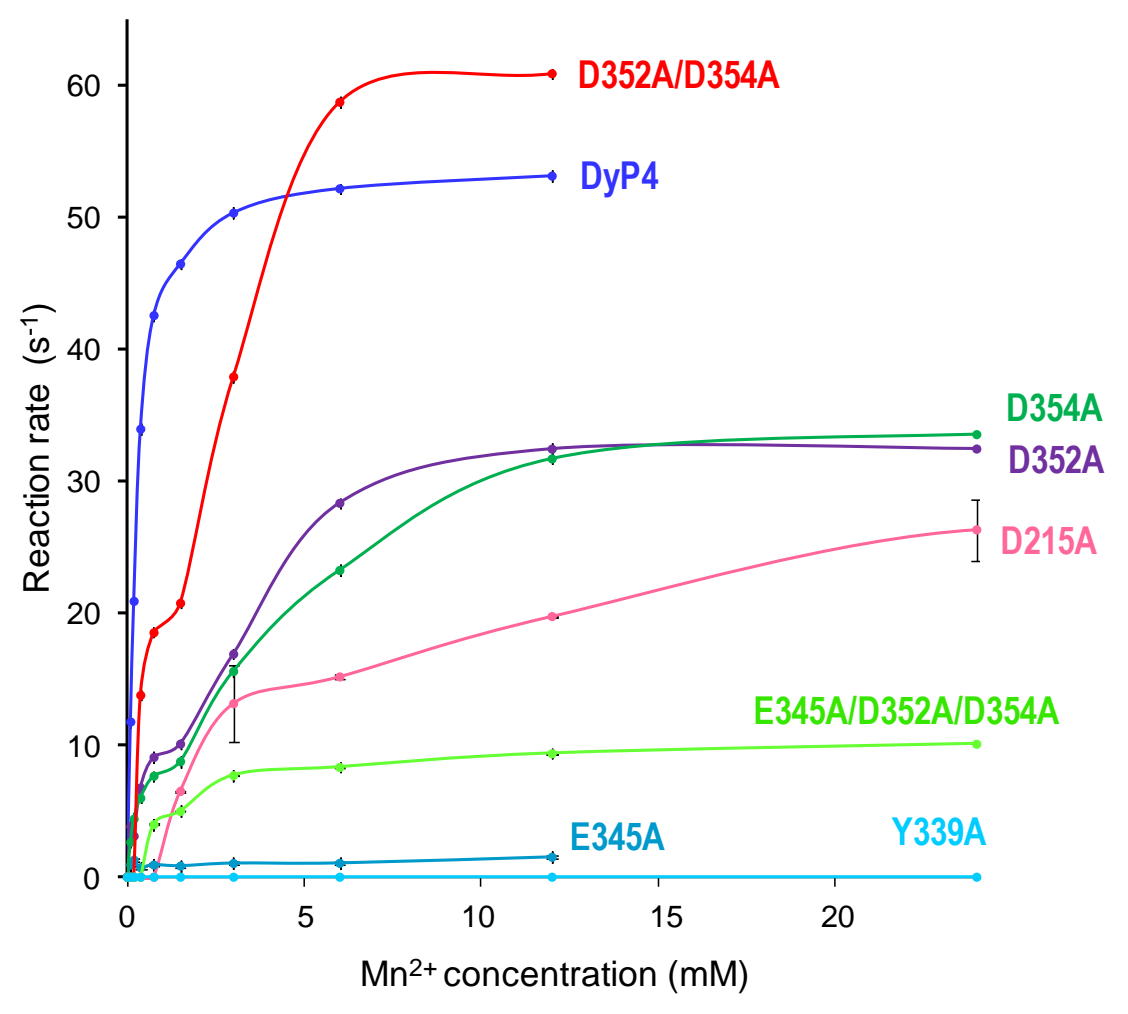

Figure S9. Kinetics of $\mathrm{Mn}^{2+}$ oxidation by PosDyP4 and seven simple, double and triple variants, with substrate concentration in arithmetic scale. See Figure 7 for representation of the same data with substrate concentration in logarithmic scale for better showing the biphasic kinetics with some of the variants. Means and 95\% confidence limits. 

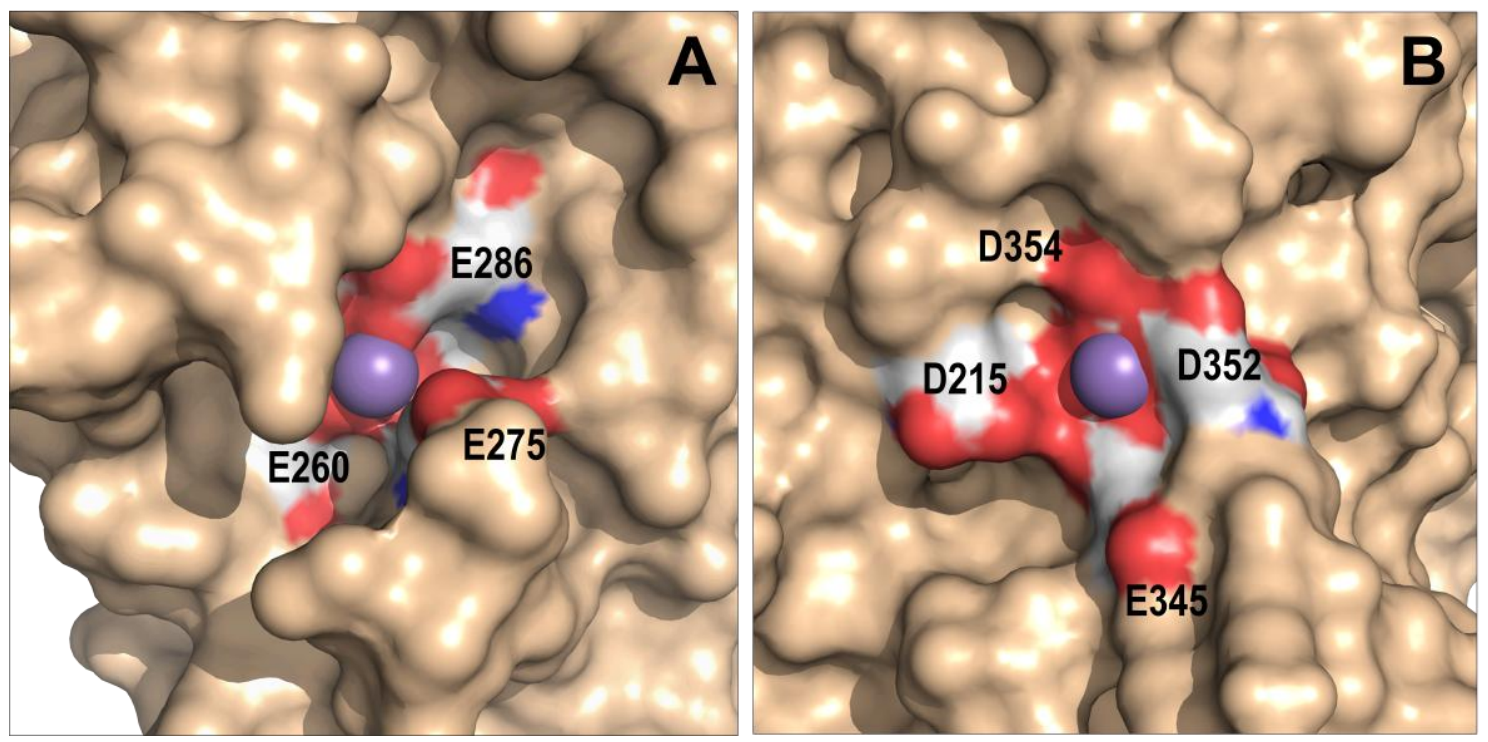

Figure S10. Solvent access surface of Amycolatopsis sp DyP2 (A) and P. ostreatus DyP4 (B) showing the exposed $\mathrm{Mn}^{2+}$ (purple sphere) oxidation site and the acidic residues involved in metal ion binding. See Figure 8 for PDB entries. 